\title{
Recent advances in EAST physics experiments in support of steady-state operation for
} ITER and CFETR

Wan, B.N.; Liang, Y.; Gong, X.Z.; Xiang, N.; Xu, G.S.; Sun, Y.; Wang, L.; Qian, J.P.; Liu, H.Q.; Zeng, L. Total number of authors:

32

Published in:

Nuclear Fusion

Link to article, DOI:

$10.1088 / 1741-4326 / \mathrm{ab} 0396$

Publication date:

2019

Document Version

Peer reviewed version

Link back to DTU Orbit

Citation (APA):

Wan, B. N., Liang, Y., Gong, X. Z., Xiang, N., Xu, G. S., Sun, Y., Wang, L., Qian, J. P., Liu, H. Q., Zeng, L., Zhang, L., Zhang, X. J., Ding, B. J., Zang, Q., Lyu, B., Garofalo, A. M., Ekedahl, A., Li, M. H., Ding, F., ... EAST Team (2019). Recent advances in EAST physics experiments in support of steady-state operation for ITER and CFETR. Nuclear Fusion, 59(11), [112003]. https://doi.org/10.1088/1741-4326/ab0396

\section{General rights}

Copyright and moral rights for the publications made accessible in the public portal are retained by the authors and/or other copyright owners and it is a condition of accessing publications that users recognise and abide by the legal requirements associated with these rights.

- Users may download and print one copy of any publication from the public portal for the purpose of private study or research.

- You may not further distribute the material or use it for any profit-making activity or commercial gain

- You may freely distribute the URL identifying the publication in the public portal 


\section{Recent advances in EAST physics experiments in support of steady- state operation for ITER and CFETR}

B. N. Wan ${ }^{1}$, Y. Liang ${ }^{1,2}$, X. Z. Gong ${ }^{1}$, N. Xiang ${ }^{1}$, G. S. Xu' ${ }^{1}$, Y. Sun ${ }^{1}$, L. Wang ${ }^{1}$, J. P. Qian ${ }^{1}$, H. Q. Liu ${ }^{1}$, L. Zeng ${ }^{1}$, L. Zhang ${ }^{1}$, X. J. Zhang ${ }^{1}$, B. J. Ding ${ }^{1}$, Q. Zang ${ }^{1}$, B. Lyu ${ }^{1}$, A.M. GAROFALO ${ }^{3}$, A. EKEDAHL ${ }^{4}$,

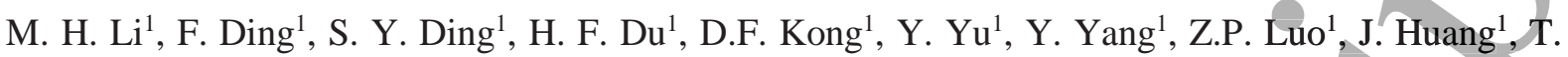
Zhang ${ }^{1}$, Y. Zhang ${ }^{1}$, G. Q. $\mathrm{Li}^{1}$, T. Y. Xia ${ }^{1}$, the EAST team** and Collaborators**

${ }^{1}$ Institute of Plasma Physics, Chinese Academy of Sciences, Hefei 230031, China

${ }^{2}$ Forschungszentrum Jülich GmbH, Institute of Energy and Climate Research -Plasma Physics (IEK-4), Association EURATOM-FZJ, 52425 Jülich, Germany

${ }^{3}$ General Atomics San Diego, California, USA

${ }^{4}$ CEA, IRFM Saint-Paul-lez-Durance, France

Contact Email: Y. Liang@ipp.cas.cn

**See Appendix

\section{Abstract}

Since the last IAEA-FEC in 2016, the EAST physics experiments have been developed further in support of high-performance steady-state operation for ITER and CFETR. First demonstration of $>100$ seconds time scale long-pulse steady-state scenario with a good plasma performance $\left(\mathrm{H}_{98(\mathrm{y} 2)} \sim 1.1\right)$ and a good control of impurity and heat exhaust with the upper tungsten divertor has been achieved on EAST using the pure radio frequency (RF) power heating and current drive. The EAST operational domain has been significantly extended towards more ITER and CFETR related high beta steady-state regime $\left(\beta_{\mathrm{P}} \sim 2.5 \& \beta_{\mathrm{N}} \sim 1.9\right.$ of using RF \& NB and $\beta_{\mathrm{P}} \sim 1.9 \& \beta_{\mathrm{N}} \sim 1.5$ of using pure RF). A large bootstrap current fraction up to $47 \%$ has been achieved with with q95 6.0-7.0. The interaction effect between the electron cyclotron resonant heating (ECRH) and two lower hybrid wave (LHW) systems has been investigated systematically, and applied for the improvement of current drive efficiency and plasma confinement quality in the steady-state scenario development on EAST. Full ELM suppression using the $n=2$ RMPs has been achieved in ITER-like standard type-I ELMy Hmode plasmas with a range of the edge safety factor of $q_{95} \approx 3.2-3.7$ on EAST. Reduction of the peak heat flux on the divertor was demonstrated using the active radiation feedback control. An increase in the total heating power and improvement of the plasma confinement are 
expected using a 0-D model prediction for higher bootstrap fraction. Towards long-pulse, high bootstrap current fraction operation, a new lower ITER-like tungsten divertor with active water-cooling will be installed, together with further increase and improvement of heating ând current drive capability. 


\section{Introduction}

As a long-term research programme of superconducting tokamaks[1][2][3][4], EAST(major radius $R \leq 1.9 \mathrm{~m}$, minor radius $a \leq 0.45 \mathrm{~m}$, plasma current $I_{p} \leq 1 \mathrm{MA}$, toroidal $B_{T} \leq 3.5 \mathrm{~T}$ ) aims to provide a suitable platform to address physics and technology issues relevant to steady-state advanced high-performance H-mode plasmas with ITER-like configuration, plasma control and heating schemes [5]. To reach this goal, EAST has equipped the continuous wave of lower hybrid current drive systems: 2.45GHz (4MW)/4.6GHz (6MW) klystron pôwer, electron cyclotron heating system:140GHz (2MW) gyrotron power, ion cyclotron resonant frequency system: $27 \mathrm{MHz}-80 \mathrm{MHz}(12 \mathrm{MW})$ generator power and the balanced neutral beam injection (NBI) systems: the 2 co-current \& 2 counter-current NBI sources (80keV/4 MW). In the past few years, EAST has been upgraded with an ITER-like active water-cooling tungsten divertor, and it is capable to handle a power load up to $10 \mathrm{MW} / \mathrm{m}^{2}$ for a long-pulse steady-state operation with high power injection. Therefore, the experience and understanding in high-performance long-pulse operation on EAST will be extremely yaluable for the next generation fusion reactors, i.e. ITER and CFETR.

In this paper, recent EAST experimental results since the $26^{\text {th }}$ IAEA Fusion Energy Conference (FEC) in 2016 are presented with the emphasis on the high normalized poloidal beta $\left(\beta_{\mathrm{P}}\right)$ scenario development and key physics related to the advanced high-performance steady-state H-mode plasmas. The recent achievements of long pulse-operation and extension of the EAST operational regime âre discussed in section 2. The physics progress in support of ITER and CFETR steady-state high performance operation is presented in section 3. A discussion of the future prospect of high bootstrap current fraction on EAST is shown in section 4. A future plan of the EAST program is descried in section 5. 


\section{Extension of steady-state operational regime with dominant RF H\&CD}

Demonstration of high performance steady-state H-mode operation with a reactor-like metal wall, a low momentum input, and electron dominated heating scheme is a critical step on the path towards the success of economical fusion energy. In the EAST superconducting tokamak, several key technical challenges related to the development of high performance steady-state H-mode operation including RF power coupling, RF heating accessibility, non-inductive current drive in high-density H-mode plasmas with deuterium as the working gas, have been investigated. A series of important breakthrough in frontier physical topics including access and sustainment of H-mode plasmas and mitigation of transient heat load associated with EdgeLocalized-Modes (ELMs) are addressed [6-9].

A repeatable and stable hundred-second time scale long-pulse steady-state scenario with a good plasma performance $\left(\mathrm{H}_{98(\mathrm{y} 2)} \sim 1.1\right)$ and a goód control of impurity and heat exhaust with the tungsten divertor has been successfully achieved on EAST using the RF power heating and current drive (H\&CD) with a total of $\sim 0.5 \mathrm{MW}$ LHW at 2.45 GHz, 1.7 MW LHW at 4.6 GHz, 0.4 MW ECH and 0.5 MW ICRF [10]. This steady-state scenario as shown in fig.1 was characterized with fully non-inductive current drive and high-frequency small-amplitude edge localized modes (ELMs), and it verified the stable control capability of heat and particle exhausts using the ITER-like tungsten divertor in hundred-second level. Plasma parameters are as follows: plasma current $I_{p}=0.4 \mathrm{MA}$, normalized poloidal beta $\left(\beta_{\mathrm{P}}\right) \sim 1.2$ toroidal magnetic field $B_{T}=2.5 \mathrm{~T}$, upper single null with the elongation $k=1.6$, the safety factor at the $95 \%$ normalized poloidal flux surface q95 6.6. This long-pulse discharge reaches wall thermal and particle equilibration[11], with the steady-state peak heat flux on the divertor plates being maintained at $\sim 3.3 \mathrm{MWm}^{-2}$ and the particle exhaust rate being maintained at $\sim 6.6 \times 10^{20} \mathrm{D} / \mathrm{s}$. It should be noted that a gradual increase of loop voltage after 90s causes by the ECRH protection of the cut-off, which suggests that ECH has the effect on the avoidance of impurity 
accumulation. The maximum tungsten surface temperature monitored by the IR camera shows that the temperature raises quickly in several seconds and reaches a stable value, $\sim 500^{\circ} \mathrm{C}$, which suggests the EAST tungsten divertor with a good power handling capability.

To achieve a high RF input power with good plasma-wave coupling efficiency, optimization of the plasma shape and the local gas puffing in front of the lower hybrid wave (LHW) antenna has been performed on EAST. It is found that the LHW-induced hot spots on the protection limiter of the LHW antenna, which limits very often the maximal LHW injection power and the duration of a long-pulse operation, can be avoided or mitigated by adjusting the plasma outer gap. Both the LHW accessibility and the current drive efficiency are sensitive to the global operational parameters, such as the toroidal magnetic field $\mathrm{B}_{\mathrm{T}}$ and the line-averaged electron density $<\mathrm{n}_{\mathrm{e}}>$. An optimized operational window for higher current drive efficiency of LHW has been identified in support of the high performance steady-state scenario development on EAST. The on-axis ECRH was applied for electron heating and the avoidance of high-Z impurity accumulation. A peaked electron temperature profile has been observed during the application of the on-axis ECRH, and the electron thermal diffusivity calculated by the power balance analysis indicates the improved confinement at the plasma core as shown in figure 2 .

More recently, experimental explorations of high $\beta_{\mathrm{P}}$ scenario for the demonstration of high bootstrap current fraction long-pulse H-mode operation capability on EAST are performed with the installation of the new LHW guide limiter to avoid hot spot issue and the use of the second ECRH system. A summary plot of $\beta_{\mathrm{P}}$ versus line-averaged density $\left(<\mathrm{n}_{\mathrm{e}}>\right)$ is shown in figure 3 for both pure RF and the combined RF and neutral beam injection (NBI) discharges. Significant extension of the operational domain of $\beta_{\mathrm{P}}$ and electron density towards the high performance regime is achieved with a range of $q_{95}$ from 6.0 to 7.0. Two typical plasma waveforms of the EAST high $\beta_{\mathrm{P}}$ scenario are shown in figure 4 . The H-mode plasma with plasma current $I_{p}=$ 
0.4MA, toroidal field $B_{T}=2.5 \mathrm{~T}$, edge safety factor $q_{95} \sim 6.8$, is successfully sustained with a high beta $\left(\beta_{\mathrm{P}} \sim 1.9\right.$, normalized beta $\left.\beta_{\mathrm{N}} \sim 1.5\right)$ at the high density regime $\left(<\mathrm{ne}>/ \mathrm{n}_{\mathrm{GW}} \sim 0.80\right)$ for 24s ( 40 times current relaxation time) (figure 4 left), where $\mathrm{n}_{\mathrm{GW}}$ is the Greenwald density limit. A total of $\sim 4 \mathrm{MW}$ RF power was applied for H\&CD. A very low loop voltage of $\sim 0.005 \mathrm{~V}$ was obtained. No sawtooth actives were observed during the whole discharge which is consistent with the measured $q$ profile $\left(q_{\min }>1.0\right)$, where the minimum $q, q_{\min }$, is above 1 . Here, the $q$ profile was measured by using the external magnetic measurements and the POlarimeterINTerferometer (POINT) constraints [12]. Transport analysis shows that a high bootstrap current fraction $\mathrm{f}_{\mathrm{bs}}$ of $\sim 45 \%$ has been achieved, and it can be stably maintained in the EAST high $\beta_{\mathrm{p}}$ scenario.

On EAST, a higher plasma beta $\left(\beta_{\mathrm{P}} \sim 2.5\right.$ and $\left.\beta_{\mathrm{N}} \sim 1.9\right)$ for a period of 8 s has been also achieved when both co- and ctr-Ip NBI were applied. The experiments have been carried out with the conventional 10s setting since the EAST NBI cannot sustain long-pulse operation at a high beam voltage $\left(V_{\text {beam }}>60 \mathrm{kV}\right)$. It should be stressed here that high density $\left(<\mathrm{n}_{\mathrm{e}}>=4.0-5.0\right.$ $\times 10^{19} / \mathrm{m}^{3}$ ) was routinely used for those discharges using NBI to avoid strong shine-through loss [13].

In addition to the exploration of the high $\beta_{\mathrm{P}}$ scenarios, extensive experiments of high $\beta_{\mathrm{N}}$ scenario development have been carried out on EAST. Figure 5 shows an example of the high $\beta_{\mathrm{N}}$ plasma discharge $\left(\mathrm{Ip}=400-500 \mathrm{kA}, B_{T}=1.5-1.6 \mathrm{~T}, q_{95}=3.4-4.4\right)$ with the ITER-like tungsten divertor. In this high $\beta_{\mathrm{N}}$ experiment, the plasma density increases up to $5.5 \times 10^{19} \mathrm{~m}^{-3}$ (Greenwald factor up to 0.75 ), and a high $\beta_{\mathrm{N}}$ of 2.1 has been obtained with a good plasma confinement $\left(\mathrm{H}_{98}(\mathrm{y} 2)=1.1\right)$. The operation domain of this scenario is shown in figure 6 . The value of $\beta_{\mathrm{N}}$ /reaches above $3 \times l_{i}$, where $l_{i}$ is the internal inductance calculated from the equilibrium analysis. By comparing the EAST results with the advanced inductive scenario 
database [14] from DIII-D, JT-60U, JET and ASDEX-U, the EAST high $\beta_{\mathrm{N}}$ scenario is still in the heating power limited regime, rather than the MHD limited regime as indicated by the $4 \times l_{i}$ line. This is supported by the fact that no clear NTM has been observed in this scenario.

In these high $\beta_{\mathrm{N}}$ scenario $\mathrm{H}$-mode plasmas, the internal transport barrier (ITB) has been often observed after step-up of the NBI power as shown in figure 7. It is rather important to note that the ITB can be obtained on EAST with various different types of plasma current profiles, including monotonic, central flat $(q(0) \sim 1)$ and reversed shear current profiles [15]. The MHD instabilities associated with these different types of current profiles have been studied. It is found that the fishbone mode $(m / n=1 / 1)$ can be beneficial to sustain the central flat $(q(0) \sim 1)$ q profile, thus a stable ITB can be obtained. The reverse-sheared Alfvén eigenmodes (RSAEs) have been observed in the reverse sheared plasma with å transient ITB formation. Recently, all these three ITB operational regimes have been further extended in the EAST 2018 campaign. The role of the plasma current profile on the formation of the ITB will be further investigated. In particular, the non-inductive current fraction in the central flat $(\mathrm{q}(0) \sim 1) \mathrm{q}$ profile plasma is larger than $40 \%$. Further investigation of this operation regime might be important for the development of the hybrid scenario for ITER and CFETR. 


\section{Progress on physics studies in support of steady-state operation for ITER and CFETR operation}

Physics studies on EAST are continued to figure out the critical issues in supporting of the high performance long-pulse steady-state operation with RF heating and current drive. In this section, several new approaches on the ITER and CFETR relevant key physics issues are highlighted.

\subsection{Heating and current drive}

\subsubsection{Effects of parametric instability}

Being an effective non-inductive method with high current drive (CD) efficiency, the lower hybrid current drive (LHCD) can be also exploited as a tool for active control of plasma current profile. Parametric instability (PI) is a non-linear interaction between radio-frequency RF waves and plasma [16], which have been observed in many LH experiments such as Alcator C-Mod [17], Tore Supra[18], FTU [19] and also EAST[20]. PI is known to excite the LH waves that has a relatively high parallel refractive index $\left(N_{/ /}\right)$[21], which can be Landau damped at low temperatures with low CD efficiency in the outer plasma region. In EAST, new experiments with $2.45 \mathrm{GHz}$ and $4.6 \mathrm{GHz} \mathrm{LH}$ waves are performed by scanning plasma density to demonstrate the effect of PI on plasma current profile in the edge region. The spectrum measurements show that the PI behaviour observed in the $2.45 \mathrm{GHz}$ case is stronger than that in the 4.6 GHz case, especially at higher density (shown in figure 8). Although the spectral broadening increases with increasing density in both cases, the increment of spectral broadening in the $2.45 \mathrm{GHz}$ case is larger than that in the $4.6 \mathrm{GHz}$ case at high density, documenting the stronger occurrence of the non-linear decay of the pump wave, which may be responsible for the loss of CD efficiency. Simultaneously, the plasma current density in the edge region ( $r / a>0.8)$ obtained from equilibrium reconstruction using an EFIT code constrained by the measurements with the external magnetic coils and POINT diagnostic was 
increased with a reduction in the source frequency or with the increase in plasma density as shown in figure 8 . So, it can be concluded that the plasma current profile modification by LHCD in the edge region shows well correlation with PI activities. It is worth mentioning that the PONIT measurements mainly focus on the core plasma and the uncertainty in the edge region is difficult to estimate at present since no direct measurement is available for the reference. However, the obtained relative trend in the edge current profile constrained by magnetic measurements is reliable. Figure 9 shows a link between the degradation of CD efficiency and the PI induced spectral broadening. It indicates that the spectral broadening has a negative and significant effect on CD efficiency for both of two LHWs on EAST. PI modeling results show that the ion-sound quasi-mode-driven PI effect cannot fully account for the loss of CD efficiency. These novel results are significant in that they give insight for the first time into how nonlinear wave-plasma interactions such as PI may directly impact the edge current profile, the control of which is critical in order to achieve optimized modes of operation in a steady-state fusion reactor.

\subsubsection{Interaction effect between ECRH and LHW}

In EAST, the interaction between ECRH and LHW has been investigated. A significant performance degradation in an electron heating dominant $\mathrm{H}$-mode plasma was observed after ECRH termination[22] (shown in figure 10). This performance degradation is accompanied by a slow decrease of $l_{i}$. The energy confinement enhancement factor $H_{98(y, 2)}$ decreases from 1.15 to 0.78 in $2.6 \mathrm{~s}$ after ECRH termination, and the internal inductance drops following the stored energy with some delay. Line averaged electron density is kept as constant during this period. The stable surface loop voltage suggests that the total non-inductive current is not changed very much. 
The analysis using GENRAY and CQL3D code shows that both the LHW electron heating and current drive move from plasma core to large radius after turning off ECRH (see in figure 11). It should be noted that the total LHW electron heating power and driven current are almost unchanged. In other words, with the early on-axis heating of ECRH before the plasma current plateau, LHW deposited more power near the plasma centre. Thus, the driven current also peaked in the core. So, from this point of view, heating of ECRH provides a way to control the LHW power deposition and also the total plasma current profile, which is crucial for the ITB formation in plasma.

\subsection{Pedestal stability}

\subsubsection{Small ELMy regime}

A highly reproducible stationary grassy ELM regime has been achieved in the EAST superconducting tokamak with water-cooled tungsten upper divertor and molybdenum first wall, exhibiting good energy confinement ( $\mathrm{H}_{98 \mathrm{y} 2}$ up to 1.4$)$, strong tungsten impurity exhaust capability, and compatibility with low rotation, hỉgh density (up to $\sim 1.1 \mathrm{n}_{\mathrm{Gw}}$ ), radiative divertor and fully non-inductive operations. Figure 12 shows statistics of ELM frequency of H-mode discharges on EAST in 2016-2018 with the plasma stored energy $\mathrm{W}_{\mathrm{p}}>120 \mathrm{~kJ}$. The ELM size generally decreases with increasing ELM frequency, $\mathrm{f}_{\mathrm{ELM}}$. The grassy ELM regime has been obtained with both $\mathrm{B}_{\mathrm{t}}$ directions. The statistics indicate that the most sensitive parameters for the grassy ELM regime access is $q_{95}$ and $\beta_{\mathrm{p}}$. The lower boundaries of the regime access for $\mathrm{f}_{\mathrm{ELM}}>0.5 \mathrm{kHz}$ is $\mathrm{q}_{95} \geq 5.3, \beta_{\mathrm{P}} \geq 1.1$ and $\mathrm{n}_{\mathrm{e}} / \mathrm{n}_{\mathrm{GW}} \geq 0.46$. $\beta_{\mathrm{N}}$ is up to 2 , limited by the total heating power currently available. This parameter space overlaps with that of the projected baseline scenario of CFETR. Higher q95, $\beta_{\mathrm{p}}$ and upper triangularity $\delta_{\mathrm{u}}$ appear to facilitate the access to higher ELM frequency, which is consistent with the JT-60U grassy-ELM prescription[23]. Although the access parameter space is similar to that of JT-60U in terms of q95, $\beta_{\mathrm{p}}$ and $\delta$, it 


\subsubsection{Type-I ELM control}

ELM suppression using resonant magnetic perturbations (RMPs) has been extended recently to low $q_{95}(\approx 3.2-3.7)$ and high beta $\left(\beta_{\mathrm{N}} \approx 1.5-2\right)$ standard type-I ELMy H-mode operational window in the summer campaign in 2018 in EAST. Here the auxiliary heating power in this experiment in EAST includes 2.5MW NBI and 1MW LHCD. Limited by the available operational window in previous experiments in EAST, ELM suppression or strong mitigation was only achieved previously in EAST with $n=1$ and 2 RMPs in a relatively high $q_{95}(\geq 5)$ and low beta $\left(\beta_{\mathrm{N}} \leq 1\right)[25][26]$. Plasma stored energy often decreases due to strong density pump out after ELM suppressed with low $n$ RMP in previous experiments. Recently, full ELM suppression is achieved by all $n=2-4$ RMPs in this new standard type-I ELMy H-mode operational window. ELM suppression with $n=3$ and 4 shows a relative minor change of stored 
energy, although strong density pumps out also occurs during this process. Ion temperature increases a lot after ELM suppression compensated the drop of energy due to density pump out. This is similar to the observations of recovery of plasma confinement after ELM suppression in DIII-D[27]. Like the observations in DIII-D[28], the ELM suppression window for $n=3$ is quite narrow. However, a large $q_{95}$ window for ELM suppression has been achieved by using the $n=2$ RMP in a similar target plasma mentioned above. Figure 13 shows that full ELM suppression was sustained during the ramp down of $q_{95}$ (via ramp up of plasma current) started from different levels. This covers a $q_{95}$ window from 3.2 to 4.2 . It demonstrated an effective ELM suppression with $n=2$ RMP in standard H mode operational window in EAST.

The maximal resonance in plasma response field modelled by linear MHD code MARS-F agrees with the optimal phasing for ELM control dưring the, scan the phasing (the phase difference between the upper and lower coil current) [26]. Recently, a multi-modal plasma response to applied non-axisymmetric fields has been found in EAST tokamak plasmas. The signature of the multi-modal response is the magnetic polarization (ratio of radial and poloidal components) of the plasma response field measured on the low field side device mid-plane, which is reproduced by GPEC modelling.

Controlling the steady-state particle and heat flux impinging on the plasma facing components is still necessary when the transient power loads induced by ELMs have been eliminated by RMPs. This is especially true for long-pulse operation. One promising solution is to use the rotating perturbed field, which has been tested in EAST [29]. The particle flux patterns on the divertor targets change synchronously with both rotating and phasing RMP fields as predicted by the modelled magnetic footprint patterns. Experiments using mixed toroidal harmonic RMPs with a static $n=3$ and a rotating $n=2$ harmonics have validated predictions that divertor 
heat and particle flux can be dynamically controlled while maintaining ELM suppression in both DIII-D and EAST[30].

\subsubsection{Impact of the $E_{r} \times B$ flow shear on ballooning-driven ELM}

The theoretical works predict that $\mathrm{E}_{\mathrm{r}} \times \mathrm{B}$ shear can affect the magnitude and evolution of the cross phase of the velocity and pressure fluctuations in the peeling-ballooning-mode-driven heat flux [31]. By using the specific co-NBI and ctr-NBI systems on EAST, an alternating $E_{r} \times B$ flow shear discharge has been performed to study the impact of the $E_{r} \times B$ flow shear on ballooning-driven ELM at a fixed high collisionallity $\left(v^{*}=2.3\right)$ [32]. The collisionallity was kept the same by the density feedback with the super molecular beam injection (SMBI) and well matching of the injecting power of co-NBI and ctr-NBI.

The H-mode plasmas are achieved in a low-recycling regime due to extensive lithium wall coating, with the combined LHW and ICRF hydrogen minority heating, at a power of $\mathrm{P}_{\mathrm{LHW}, 4.6 \mathrm{GHz}}=1.5 \mathrm{MW}, \mathrm{P}_{\mathrm{LHW}, 2.45 \mathrm{GHz}}=0.5 \mathrm{MW}$. Deposition of ICRF is at the center of deuterium plasmas. After the L-H mode transition, the H-mode plasma are modulated by periodically alternating the direction of NBI, either co-NBI or ctr-NBI with $\mathrm{P}_{\mathrm{co}-\mathrm{NBI}}=0.4 \mathrm{MW}$ and $\mathrm{P}_{\mathrm{ctr}-\mathrm{NBI}}=0.5 \mathrm{MW}$, respectively, as shown in figure $14(\mathrm{c})$. With the alternating of the coNBI and ctr-NBI, the velocity of the toroidal rotation in plasma centre is changed periodically from $\sim 50 \mathrm{~km} / \mathrm{s}(\mathrm{co}-\mathrm{NBI})$ to $\sim-10 \mathrm{~km} / \mathrm{s}$ (ctr-NBI), as illustrated as the red-dash line in Figure14 (d).

Figure 15 illustrates the profiles of Doppler frequency $\mathrm{f}_{\text {Doppler }}$ measured from the Doppler Backscatter System (DBS) on EAST, here the radial electric field $\mathrm{E}_{\mathrm{r}}$ is proportional to $\mathrm{f}_{\text {Doppler }}$ for $\mathrm{E}_{\mathrm{r}}=-\mathrm{B} * \mathrm{u}_{\perp}=-\frac{2 \pi \mathrm{B}}{\mathrm{k}_{\perp}} \mathrm{f}_{\text {Doppler }} \propto \mathrm{f}_{\text {Doppler }}$ with a fixed launch angle of DBS. It can be found that the Doppler frequency $f_{\text {Doppler }}$ wells in the pedestal region show big differences upon periodically altering the direction of NBI. The well becomes more negative 
at the ctr-NBI case. The maximum value of $\left|E_{r}\right| \sim 6.2 \mathrm{kV} / \mathrm{m}$ at the bottom of $\mathrm{E}_{\mathrm{r}}$ well. As shown in Figure $1(\mathrm{~d})$, the toroidal rotation changed from $\sim 50 \mathrm{~km} / \mathrm{s}$ to $\sim-10 \mathrm{~km} / \mathrm{s}$ after the counter neutral beam injection, which contributed the negative radial electric field in the ion force balance equation $E_{r}=\frac{1}{Z_{i} e_{i}} \frac{\partial P_{i}}{\partial r}-v_{\theta} B_{t}+v_{t} B_{\theta}$. Here, the $v_{t}$ is the velocity of toroidal rotation. The ELMs are suppressed by $\sim 80 \%$ at the ctr-NBI case with the maximal $\left|\mathrm{E}_{\mathrm{r}}\right|$ increased by a factor of $\sim 2.7$.

The results reveal that the increased $\mathrm{E}_{\mathrm{r}} \times \mathrm{B}$ flow shear can significantly mitigate the ELM, or even totally suppress the ELM when the shear is large enough. Our simulations with BOUT++ support the observations on EAST, and further indicates that the increased $\mathrm{E}_{\mathrm{r}} \times \mathrm{B}$ can both reduce the linear growth rate of ballooning mode and shorten its growth time (phase coherence time, PCT). The enhanced nonlinear interactions shorten the PCT of ballooning mode, which is validated by the bispectrum study on EAST. All those studies suggest a new way to control the ELM.

\subsection{Power and particle exhaust}

\subsubsection{High Z impurity control}

It has been widely accepted that tungsten (W) will be used in ITER divertor, and it is the top candidate plasma facing material for DEMO and CFETR. On EAST, it is often observed that the long-pulse steady-state $\mathrm{H}$-mode is restricted by largely increased radiated power in plasma core due to the tungsten accumulation [33]. Tungsten control is therefore a crucial issue for the EAST long-pulse H-mode operation. A dedicated experiment of high Z impurity accumulation avoidance (discharge \#73886) has been performed on EAST by applying the on-axis ECRH during the H-mode phase as shown in Fig. 16. In this experiment, the power of ECRH is deposited at $\rho<0.1$. After the ECRH is turned off at $t=36.5 \mathrm{~s}$, the high-Z impurity of $\mathrm{W}$ build 
up quickly, thus a steady-state H-mode could not be sustained. A comparison of density profiles of $\mathrm{W}^{45^{+}}$measured with and without ECRH is shown in Figure 16. The result indicates the $\mathrm{W}^{45^{+}}$ ion is dramatically pumped out from plasma core with ECRH. The maximal density of the $\mathrm{W}^{45+}$ ion, $\mathrm{nw}_{\mathrm{w}}{ }^{45+}$, decrease from 3.5 to $1.9 \times 10^{8} \mathrm{~cm}^{-3}$, and its peak deposition moves outward from $\rho=0.13$ to 0.2. In recent EAST long-pulse H-mode operation, the on-axis ECRH has been routinely superimposed on the LHW and ICRH sustained H-mode phase to avoid the high-Z impurity accumulation and control high-Z impurity content.

\subsubsection{Radiation feedback control}

Impurity seeding has been recognized as an attractive method for the steady-state heat flux control in a long-pulse high power H-mode operation, especially for superconducting tokamaks like EAST, ITER and CFETR. The seeding impurities can dissipate a large fraction of the thermal energy into radiation, and thus reduce the peak heat flux and total power incident on the divertor target plates. The active feedback control of radiation power and thus heat load towards long-pulse operation has been developed and successfully achieved in EAST using neon (Ne) impurity seeding [34]. By seeding a sequence of short neon impurity pulses with the SMBI from the outer mid-plane, the plasma radiation power can be well controlled. Reliable control of the total radiated power of the bulk plasma has been successfully achieved in longpulse upper single null (USN) discharges with a tungsten divertor. The achieved control range of $f_{\text {rad }}$ is $20 \%-30 \%$ in L-mode regimes and $18 \%-36 \%$ in H-mode regimes, where $f_{\text {rad }}$ is the radiation fraction with respect to the total injected power. The temperature of the divertor target plates was maintained at a low level due to increased power during the radiative control phase. The peak particle flux on the divertor target was decreased by feedforward Ne injection in the L-mode discharges, while the Ne pulses from the SMBI had no influence on the peak particle flux because of the very small amount of injected Ne particles. Figure 17 shows the control results for a serial of sequent long-pulse H-mode discharges. During the entire duration of the 
feedback control phase, the temperature of the divertor target plates is maintained constant, however, it starts to increases immediately after the feedback control was turned off. At the strike point of the outer target plates, the temperature descends around 250 - $300 \mathrm{~K}$ during the feedback control phase, which suggests that the heat flux incident on the divertor target is well reduced. In addition, the simulations on the edge impurity transport and radiation using SOLPS code have been carried out with different seeding impurity species, and the results have been applied for optimization of the radiation feedback control in EAST [35]. In the 2018 campaign, the radiation feedback control with neon seeding from divertor region was successfully extended in the small ELMy regime [36]. The neon seeding from divertor region also exhibits a great success for detachment feedback control [11].

\subsubsection{Recycling and particle exhaust}

Fuel recycling strongly affects plasma density and confinement performance, especially in the high power long pulse plasma operation[33,37]. In EAST, the first wall baking and alternate $\mathrm{D}_{2} /$ He glow discharge cleaning of up to $\sim 1$ month is employed to reduce impurity and hydrogen content in the vacuum vessel and first wall surface, and an ultimate vacuum of $3.6 \times 10^{-6} \mathrm{~Pa}$ is achieved after long time wall conditioning, which provides a good wall condition for the plasma operation. Fuel recycling is usually very high in the initial plasma operation, and it's decreased gradually along with discharges. Moreover, low-Z material of silicon and lithium coating on the first wall is effective to control fuel recycling, and lithium is proven to be more effective than silicon, and lithium coating assisted with ICRF discharge cleaning is a routine wall conditioning method to control fuel recycling in EAST[38].

In EAST 2018 campaign, helical wave plasmas (HWP) are successfully excited by the RF wave power via a helical antenna, with the following parameters: $\mathrm{P}_{\mathrm{rf}}=10-30 \mathrm{~kW} @ 27 \mathrm{MHz}$, helium $\sim 0.27 \mathrm{~Pa}, \mathrm{~B}_{\mathrm{T}}=0.5-1 \mathrm{~T}$. The HWP plasmas are almost toroidally uniform, and mainly localized 
inside helical antenna in poloidal direction, as shown in figure 18(a). This is for the first time applying the HWP for conditioning the first wall under a strong magnetic field $(\sim 1 \mathrm{~T})$ in tokamaks, the retained deuterium particles are obviously desorbed during the HWP discharge cleaning, with a removal rate of $\sim 10^{19} \mathrm{D}$-atoms/s, mainly in the form of HD via isotope exchange. Moreover, Direct-Current Glow Discharge Cleaning (DC-GDC) under strong magnetic field of $2 \mathrm{~T}$ is also successfully operated in EAST tokamak in $0.5-4.5$ Pa helium atmosphere by using 1 - 4 GDC anodes with 1 - 4 A GDC current per anode, leàding to a total GDC current of $1-24$ A. The DC-GDC plasmas flow along magnetic field as shown in figure 18(b). It was considered that the GDC could not work under strong magnetic field because glow discharge current is hard to flow cross magnetic field line. However, in toroidal direction along with magnetic field line, glow discharge current could be kept between the GDC anodes and the vessel walls, this may be the main reason why the DC-GDC works stably in strong magnetic field. Both the HWP and the DC-GDC works well under strong magnetic field, providing more choices of wall conditioning in the future fusion devices with strong magnetic field.

\section{Extrapolation from EAST long pulse operation to $>\mathbf{5 0} \%$ bootstrap current fraction}

After achieving $>100 \mathrm{~s}$ long pulse $\mathrm{H}$-mode, EAST is now proposing a new milestone, to achieve $50 \%$ bootstrap current fraction at q95 comparable to those of ITER and CFETR steadystate scenarios, for its next development. Unlike the more compact conventional tokamak, EAST, the superconducting tokamak, which shares its inner space with the shielding, cryosubsystem, has relatively high aspect ratio $(\mathrm{R} / \mathrm{a}=1.85 / 0.45=4.11)$. This feature makes it more difficult in pursing high bootstrap current fraction in plasma operation due to the proportional relation between the bootstrap current fraction and the inversed aspect ratio. For example, the joint EAST/DIII-D research team developed a high confinement, high $\beta_{\mathrm{p}}$ scenario on DIII-D as one of the candidate scenarios for EAST future long-pulse high performance plasma [19]. 
This scenario achieves $\mathrm{H}_{98(\mathrm{y} 2)}>1.5$ and realizes $\mathrm{f}_{\mathrm{bs}} \sim 80 \%$ at $\beta_{\mathrm{p}} \geq 3.0$. Considering the relation, $\mathrm{fbs}_{\mathrm{bs}} \sim \varepsilon^{0.5} \times \beta_{\mathrm{p}}$, EAST will have nearly $20 \%$ lower bootstrap current in the same confinement and beta. The same 0 -D extrapolation suggests that EAST may need $\beta_{\mathrm{p}} \geq 2.5$ in order to achieve $\mathrm{f}_{\mathrm{bs}} \sim 50 \%$, which depends on collisionality as well. The fact is that in the EAST long-pulse discharges, plasma poloidal beta is only around 1.2 and the bootstrap current fraction is usually about $30 \%$ or below. There is still a large gap toward the goal of $\mathrm{f}_{\mathrm{bs}} \sim 50 \%$ in the plasma operational space. Nevertheless, the EAST team will focus on this research and break through the scope of the operational space.

A path to the goal of $\mathrm{f}_{\mathrm{bs}} \sim 50 \%$ can be illustrated in fig. 19. Based on the $0-\mathrm{D}$ modelling of EAST parameters, this figure shows the possible operational space expressed by the bootstrap current fraction, $\mathrm{H}_{98(\mathrm{y} 2)}$ and the line-average density for the plasma, which has $400 \mathrm{kA}$ of the plasma current, i.e. q95 6.5. In fig. 16, the long-pulse regime achieved in EAST 2017 campaign is highlighted in large red ellipse. To achieve the $\mathrm{f}_{\mathrm{bs}}$ target, the 0 -D simulation suggests three working directions. Firstly, enhance the effective auxiliary heating capability. In 2017 campaign, the total injected power (not absorbed power) is usually about 3-5 MW in long-pulse discharges. Additional 3-5 MW of the steady-state auxiliary heating power is expected. Otherwise, we will need to trade confinement for heating power. The regime in green ellipse can also be our goal, if the plasma can achieve very high confinement, $\mathrm{H}_{98(\mathrm{y} 2)}>1$.35. Here comes the second working direction - higher confinement (better than standard H-mode). In this way, high confinement ensures the 'economic' high performance plasma operation with relatively low input power. EAST might need 6-7 MW to achieve the bootstrap fraction target. However, the high confinement itself is very challenging. It requires substantial increase of confinement based on the standard H-mode. An ITB is usually essential in these plasmas. The third working direction is fully non-inductive plasma operation with high density. Historically, EAST relies on the lower hybrid wave heating and current drive very much, while low density is the 
favourable condition in this regime. Figure 19 suggests plasma density like $5.0 \times 10^{19} \mathrm{~m}^{-3}$ or higher should be tested in the experiment in order to pursue the bootstrap fraction target. How to improve the current drive efficiency of the lower hybrid wave becomes a very important issue in the high-density scenario. In the EAST campaign 2018, more endeavours have been made to pursue the bootstrap current target. The representative discharges are shown in stars in figure 19 , where $\sim 45 \% \mathrm{f}_{\mathrm{bs}}$ was obtained with the pure RF H\&CD. It is foreseen that the $50 \%$ $\mathrm{f}_{\mathrm{bs}}$ target is achievable with 2 extra gyrotrons (2 MW) for H\&CD in the campaign 2019.

\section{Summary and future plan}

In all, several great progresses have been made in the development and understanding of relevant physics and issues with respect to the long-pulse steady-state operation since the last IAEA FEC in 2016. The demonstration of a long-pulse steady-state H-mode of 101.2s with small ELMs and a good global performance $\left(\mathrm{H}_{98(\mathrm{y} 2)} \sim 1.1\right)$ was achieved through the integrated operation. The long-pulse discharge reaches wall thermal and particle balance with the ITERlike tungsten divertor. To demonstrate high $\beta_{\mathrm{P}}$, high $\mathrm{f}_{\mathrm{bs}}$ for ITER and CFETR, the extension of the EAST operational domain towards the higher beta regime were obtained by using different heating schemes, in which $\beta_{\mathrm{P}} \sim 2.5 \& \beta_{\mathrm{N}} \sim 1.9$ of using RF\&NB and $\beta_{\mathrm{P}} \sim 1.9 \& \beta_{\mathrm{N}} \sim$ 1.5 of using RF only. Meanwhile, the sustainment of high $\beta_{\mathrm{P}} \sim 1.9$ of using RF only with ne/n $\mathrm{n}_{\mathrm{GW}}$ $\sim 80 \%, \mathrm{f}_{\mathrm{bs}} \sim 45 \%$ at $q_{95} \sim 6.8$ for $24 \mathrm{~s}$ was achieved, which is particularly suited for high $\beta_{\mathrm{P}}$ longpulse operation. The good confinement with ITB was achieved in these plasmas. The use of on-axis ECRH was demonstrated to be effective methods to avoid the high-Z impurity accumulation for the EAST long-pulse operation. It was also shown that the interaction effect between the ECRH and two LHW systems (2.45GHz and 4.6GHz), which allows LHW to deposit more power in plasma core regime with enhanced current drive capability. A highly reproducible stationary grassy ELM regime was achieved in EAST with exhibition of good energy confinement (H98y2 up to 1.4), strong tungsten impurity exhaust capability. Full ELM 
suppression with the application of $n=2$ RMPs was achieved in the standard type-I ELMy Hmode plasmas with a window of $q_{95} \approx 3.2-3.7$ and a relative high beta $\left(\beta_{\mathrm{N}} \approx 1.5-2\right)$. Reduction of the peak heat flux on the divertor using the active radiation feedback control shows a promising method for EAST heat flux control in the long-pulse steady-state operation. Upcoming EAST experiments, the integration of techniques and physics understanding will accelerate the exploration of the EAST high performance, high bootstrap current fraction ( $\mathrm{f}_{\mathrm{bs}}$ $\geq 50 \%)$ steady-state scenario.

With the features such as electron heating dominant, low torque and ITER-like tungsten divertor, EAST made unique contributions to some critical issues of ITER and CFETR. EAST has demonstrated steady-state operations with similar $q_{95}$ and good confinement of CFETR. As shown in section 2 , discharge 81163 has $q_{95}=6.8$, good confinement and relatively high density $<$ ne $>/ \mathrm{n}_{\mathrm{GW}} \sim 0.80$. However, the $\beta_{\mathrm{N}}$ are still lower than the CFETR reference scenario[39]. More experiments need to perform to push the $\beta_{\mathrm{N}}$ up to 2.8 , which is the target $\beta_{\mathrm{N}}$ of the steady-state operation of ITER and CFETR. EAST also achieved a small ELM regime compatible with the CFETR steady-state scenario, as described in section 3.2.1. This gives a possible solution to the handle the ELM heat flux on the CFETR divertor target plate. For the power and particle exhaust, as shown in section 3.3, EAST clearly shows the tungsten impurity accumulation could be controlled by ECRH, and divertor radiation feedback control has been realized by impurity seeding, this gives more confidence to control the impurity and the heat flux on the target plates.

Towards very long-pulse, high $\mathrm{f}_{\mathrm{bs}}$ plasma operation, a further extension of the ECH system with 2 more gyrotrons is underway and will give total 4.0MW power for heating, current drive and profile control. In order to support the physical research on EAST, two optimization methods have been applied for NBI system in this summer experiment. Firstly, adjusting the 
voltage gradient on the accelerator is employed to raise the electric field in the first gap. By this method, the injected beam power is boosted about 25\%. Secondly, the technology of beam re-turn on is also developed and applied. This enables the neutral beam injection system to have the long-pulse operation ability even if there is a spark down. Meanwhile, a new ITERlike monoblock structure with $\sim 10 \mathrm{MW} / \mathrm{m}^{2}$ power handling capability (shown in fig.20) will be used in the target plates and flat-W-tile structure with $\sim 5 \mathrm{MW} / \mathrm{m}^{2}$ power handling capability will be used in the dome and baffle. The surface of end boxes (water pipe comnector) are oriented to avoid direct exposure to high heat flux. The capability of water-cooling system will be enhanced with water flow velocity increasing from 4 to $8 \mathrm{~m} / \mathrm{s}$. The installation of new W lower diveror was scheduled in 2019. 


\section{Acknowledgements}

This work was supported by National Magnetic Confinement Fusion Science Program of China under Contract Nos. 2015GB101000, 2015GB102000, 2015GB103000 $2015 G B 110005$ and National Natural Science Foundation of China under Grant No. 11261140328, 11422546,11575249 . 


\section{Appendix}

\section{The EAST Team}

Baonian Wan ${ }^{1}$, Jiangang $\mathrm{Li}^{1}$, Yuanxi Wan ${ }^{1}$, Yuntao Song ${ }^{1}$, Xiaodong Zhang ${ }^{1}$, Peng Fu ${ }^{1}$, Houyang Guo $^{1,15}$, Yunfeng Liang ${ }^{1,32}$, Xianzhu Gong ${ }^{1}$, Guosheng $\mathrm{Xu}^{1}$, Bingjia Xiao ${ }^{1}$, Yu Wu ${ }^{1}$, Xiang Gao ${ }^{1}$, Damao Yao ${ }^{1}$, Nong Xiang ${ }^{1}$, Liqun $\mathrm{Hu}^{1}$, Jiafang Shan ${ }^{1}$, Yanping Zhao ${ }^{1}$, Guangnan Luo ${ }^{1}$, Chundong $\mathrm{Hu}^{1}$, Jiefeng $\mathrm{Wu}^{1}$, Jiansheng $\mathrm{Hu}^{1}$, Biao Shen ${ }^{1}$, Zhenshan $\mathrm{Ji}^{1}$, Ge Gao ${ }^{1}$, Yiyun Huang ${ }^{1}$, Liuwei, Xu ${ }^{1}$, Qiyong Zhang ${ }^{1}$, Ming Zhuang ${ }^{1}$, Fukun Liu ${ }^{1}$, Junyu Zhao ${ }^{1}$, Junling Chen ${ }^{1}$, Bin Cao ${ }^{1}$, Lei Cáo ${ }^{1}$, Jiafeng Chang $^{1}$, Kaiyun Chen ${ }^{1}$, Ran Chen ${ }^{1}$, Yebin Chen $^{1}$, Anyi Cheng ${ }^{1}$, Yong Cheng ${ }^{1}$, Yu Dai ${ }^{1}$, Wei Deng ${ }^{1}$, Xu Deng ${ }^{1}$, Bojiang Ding ${ }^{1}$, Fang Ding ${ }^{1}$, Rui Ding ${ }^{1}$, Siye Ding ${ }^{1}$, Shijun Du ${ }^{1}$, Yanmin Duan ${ }^{1}$, Jianqiang Feng ${ }^{1}$, Kaifu Gan ${ }^{1}$, Daming Gao ${ }^{1}$, Qingsheng Gao ${ }^{1}$, Wei Gao ${ }^{1}$, Yongqi Gu${ }^{1}$, Yong Guo ${ }^{1}$, Xiaofeng $\mathrm{Han}^{1}$, Ailan $\mathrm{Hu}^{1}$, Chang $\mathrm{Hu}^{1}$, Guanghai $\mathrm{Hu}^{1}$, Huaichuan $\mathrm{Hu}^{1}$, Liangbin $\mathrm{Hu}^{1}$, Qingsheng $\mathrm{Hu}^{1}$, Yanlan $\mathrm{Hu}^{1}$, Zhenhua $\mathrm{Hu}^{1}$, Juan Huang ${ }^{1}$, Liansheng Huang ${ }^{1}$, Ming Huang ${ }^{1}$, Ronglin Huang ${ }^{1}$, Xiang Ji ${ }^{1}$, Hua $\mathrm{Jia}^{1}$, Caichao Jiang ${ }^{1}$, Yinxian $\mathrm{Jie}^{1}$, Songqing $\mathrm{Ju}^{1}$, Defeng Kong ${ }^{1}$, Changzheng $\mathrm{Li}^{1}$, Erzhong $\mathrm{Li}^{1}$, Guoqiang $\mathrm{Li}^{1}$, Jiahong Li ${ }^{1}$, Jun $\mathrm{Li}^{1}$, Junjun $\mathrm{Li}^{1}$, Miaohui Li ${ }^{1}$, Qiang $\mathrm{Li}^{1}$, Shanshan Li ${ }^{1}$, Shi Li ${ }^{1}$, Yadong $\mathrm{Li}^{1}$, Yingying $\mathrm{Li}^{1}$, Lizhen Liang${ }^{1}$, Yanchuan $\mathrm{Liao}^{1}$, Shiyao Lin ${ }^{1}$, Bili Ling ${ }^{1}$, Changle Liu ${ }^{1}$, Haiqing Liu $^{1}$, Huajun Liu ${ }^{1}$, Liang Liu ${ }^{1}$, Shaocheng Liu ${ }^{1}$, Sheng Liu ${ }^{1}$, Xiaogang Liu ${ }^{1}$, Xiaoju Liu ${ }^{1}$, Yong Liu ${ }^{1}$, Zhihong Liu ${ }^{1}$, Zhimin Liu ${ }^{1}$, Zixi Liu ${ }^{1}$, Feng Long ${ }^{1}$, Jianhua Lu ${ }^{1}$, Zhengping Luo ${ }^{1}$, Bo Lyu ${ }^{1}$, Dengkui $\mathrm{Ma}^{1}$, Lin $\mathrm{Ma}^{1}$, Huafeng $\mathrm{Mao}^{1}$, Wendong Ma${ }^{1}$, Songtao $\mathrm{Mao}^{1}$, Yuzhou Mao ${ }^{1}$, Tingfeng Ming ${ }^{1}$, Chao $\mathrm{Mo}^{1}$, Qicai Ni ${ }^{1}$, Minzhong Qi ${ }^{1}$, Chao Pan ${ }^{1}$, Chengkang Pan ${ }^{1}$, Shengmin Pan ${ }^{1}$, Jing Qian ${ }^{1}$, Jinping Qian $^{1}$, Li Qian¹, Yanda Qian¹, Chengming Qin ${ }^{1}$, Lilong Qiu ${ }^{1}$, Qilong Ren ${ }^{1}$, Zhibin Ren ${ }^{1}$, Junsong Shen $^{1}$, Linhai Sheng ${ }^{1}$, Peng Sheng ${ }^{1}$, Zhicai Sheng ${ }^{1}$, Nan Shiㄹ, Shihua Song ${ }^{1}$, Pengjun Sun ${ }^{1}$, Xiaoyang Sun ${ }^{1}$, Youwen Sun ${ }^{1}$, Jie Tang ${ }^{1}$, Ling Tao ${ }^{1}$, Ang $\mathrm{Ti}^{1}$, Erhui Wang ${ }^{1}$, Feng Wang ${ }^{1}$, Fudi Wang ${ }^{1}$, Houyin Wang ${ }^{1}$, Huazhong Wang ${ }^{1}$, Huihui Wang ${ }^{1}$, Huiqian Wang ${ }^{1}$, Jian Wang ${ }^{1}$, Lei Wang ${ }^{1}$, Liang Wang ${ }^{1}$, Linsen Wang ${ }^{1}$, Mao Wang ${ }^{1}$, Ping Wang ${ }^{1}$, Shengming Wang', Wanjing Wang ${ }^{1}$, Xiaojie Wang ${ }^{1}$, Xiaoming Wang ${ }^{1}$, Yating Wang ${ }^{1}$, Yiyun Wang ${ }^{1}$, Yong Wang ${ }^{1}$, Yumin Wang ${ }^{1}$, Jianglong Wei ${ }^{1}$, Jing $\mathrm{Wei}^{1}$, Xuechao $\mathrm{Wei}^{1}$, Bin $\mathrm{Wu}^{1}$, Dajun $\mathrm{Wu}^{1}$, Hao $\mathrm{Wu}{ }^{1}$, Jinhua $\mathrm{Wu}^{1}$, Xiangming $\mathrm{Wu}^{1}$, Yibing $\mathrm{Wu}^{1}$, Zhenwei $\mathrm{Wu}^{1}$, Zege $\mathrm{Wu}^{1}$, Weibin $\mathrm{Xi}^{1}$, Genhai Xiao ${ }^{1}$, Tianyang Xia ${ }^{1}$, Yezheng Xiao ${ }^{1}$, Hunyi Xie ${ }^{1}$,

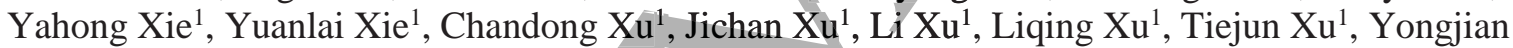
$\mathrm{Xu}^{1}$, Ning Yan ${ }^{1}$, Fei Yang ${ }^{1}$, Jianhua Yang ${ }^{1}$, Lei Yang ${ }^{1}$, Qingxi Yang ${ }^{1}$, Yao Yang ${ }^{1}$, Yong Yang ${ }^{1}$, Zhongshi Yang ${ }^{1}$, Min Yu ${ }^{1}$, Yaowei Yu ${ }^{1}$, Qiping Yuan ${ }^{1}$, Shuai Yuan ${ }^{1}$, Qing Zang ${ }^{1}$, Long Zeng ${ }^{1}$, Jizong Zhang $^{1}$, Kai Zhang ${ }^{1}$, Liyuan Zhang ${ }^{1}$, Ling Zhang ${ }^{1}$, Ruirui Zhang ${ }^{1}$, Shoubiao Zhang ${ }^{1}$, Tao Zhang ${ }^{1}$, Wei Zhang ${ }^{1}$, Xinjun Zhang ${ }^{1}$, Yang Zhang ${ }^{1}$ Zuchao Zhang ${ }^{1}$, Hailin Zhao ${ }^{1}$, Jinlong Zhao ${ }^{1}$, Fubin Zheng ${ }^{1}$, Yuanyang Zheng ${ }^{1}$, Guoqiang Zhong ${ }^{1}$, Ruijie Zhou ${ }^{1}$, Haishan Zhou ${ }^{1}$, Yue Zhou ${ }^{1}$, Dahuan Zhu ${ }^{1}$, Haisheng Zhu ${ }^{1}$, Ping Zhu ${ }^{1}$, Zeying Zhu ${ }^{1}$, Huidong Zhuang ${ }^{1}$, Zibo Zhou ${ }^{1}$, Zhiyong Zhou ${ }^{1}$, Zhiwei Zhou $^{1}$, Guizhong Zuo ${ }^{1}$.

\section{International and Domestic Collaborâtors:}

Tao Lan², Adi Liu², Wandong Liu², Hong Qin²,16, Shaojie Wang², Minyou Ye², Changxuan Yu², Yi $\mathrm{Yu}^{2}$, Ping Zhu ${ }^{2}$, Wei Chen ${ }^{3}$, Guangjiu Lei ${ }^{3}$, Lin Nie ${ }^{3}$, Xianming Song ${ }^{3}$, Min Xu ${ }^{3}$, Yuhong Xu ${ }^{3}$, Huang Yuan $^{3}$, Nanhua $\mathrm{Yao}^{3}$, Zhe Gao ${ }^{4}$, Yuhe $\mathrm{Li}^{4}$, Zhongjing Chen ${ }^{5}$, Tieshuan Fan ${ }^{5}$, Xingyu Peng ${ }^{5}$ Liu Chen $^{6,21}$, Zhiwei Ma ${ }^{6}$, Zhiyong Qiu ${ }^{6}$, Zengmao Sheng ${ }^{6}$, Yong Xiao ${ }^{6}$, Xiaogang Wang ${ }^{7}$, Zhongyong Chen $^{8}$, Yonghua Ding ${ }^{8}$, Xiwei Hu${ }^{8}$, Zijiang Wang ${ }^{8}$, Ge Zhuang ${ }^{8}$, Daming Liu ${ }^{9}$, Jiarong Luo ${ }^{9}$, Fangchuan Zhong ${ }^{9}$, Hongbin Ding ${ }^{10}$, Dezhen Wang ${ }^{10}$, Zhengxiong Wang ${ }^{10}$, Chenggang Jin ${ }^{11}$, Xuemei $\mathrm{Wu}^{11}$, Xiaofei Yang ${ }^{11}$, Jianhua Zhang ${ }^{12}$, Qingyuan $\mathrm{Hu}^{12}$, Xi Yuan ${ }^{12}$, Changqi Chen ${ }^{13}$, Shuyi Gan ${ }^{13}$, Xudi Wang ${ }^{13}$, Congzhong Wu ${ }^{13}$, Chongwei Zhang ${ }^{13}$, Ting Zhang ${ }^{13}$, Wu Zhu ${ }^{13}$, Erhua Kong ${ }^{14}$, Kaisong Wang $^{14}$, Chuanli Wang ${ }^{14}$, Hongtao Yang ${ }^{14}$, Lixiang Zhang ${ }^{14}$, Paul Anderson ${ }^{15}$, Gheni Abla ${ }^{15}$, Vincent Chan $^{15}$, John L. Doane ${ }^{15}$, Andrea Garofalo ${ }^{15}$, Punit Gohil ${ }^{15}$, Houyang Guo ${ }^{15}$, David Hill ${ }^{15}$, Chris Holcomb $^{15}$, Chung Lih Hsieh ${ }^{15}$, Ruey Hong ${ }^{15}$, David Humphreys ${ }^{15}$, Alan Walter Hyatt ${ }^{15}$, Gary Jackson $^{15}$, Egemen Kolemen ${ }^{15}$, Matthew Lanctot ${ }^{15}$, Lang Lao ${ }^{15}$, James Leuer ${ }^{15}$, John Lohr ${ }^{15}$, Mohamad Ali Mahdavi ${ }^{15}$, Robert Olstad ${ }^{15}$, Ben Penaflor ${ }^{15}$, Ron Prater ${ }^{15}$, David Piglowski ${ }^{15}$, Michael Schaffe ${ }^{15}$, Eugenio Schuster ${ }^{15}$, Tim Scoville ${ }^{15}$, Wayne Solomon ${ }^{15}$, Gary Staebler ${ }^{15}$, Mickey Wade ${ }^{15}$, Mike Walker ${ }^{15}$, Anders Welander ${ }^{15}$, Manfred Bitter ${ }^{16}$, Robert Budny ${ }^{16}$, Robert A. Ellis ${ }^{16}$, Guoyong Fu $^{16}$, Nat Fisch ${ }^{16}$, Rich Hawryluk ${ }^{16}$, Kenneth W. Hill ${ }^{16}$, Joel Hosea ${ }^{16}$, Michael A. Jaworski ${ }^{16}$, Egemen 
Kolemen ${ }^{16}$, Dennis Mansfield ${ }^{16}$, Dana M. Mastrovito ${ }^{16}$, Jonathan Menard ${ }^{16}$, Dennis Mueller ${ }^{16}$, Novmir Pablant ${ }^{16}$, Yang Ren ${ }^{16}$, Lane Roquemore ${ }^{16}$, Filippo Scotti ${ }^{16}$, Gary Taylor ${ }^{16}$, Kevin Tritz ${ }^{16}$, Randy Wilson $^{16}$, Michael Zarnstorff ${ }^{16}$, Leonid E. Zakharov ${ }^{16}$, Seung Gyou Baek ${ }^{17}$, Beck Bill ${ }^{17}$, Paul T. Bonoli $^{17}$, Robert Granetz ${ }^{17}$, Yijun Lin ${ }^{17}$, Ron Parker ${ }^{17}$, Shunichi Shiraiwa ${ }^{17}$, Josh Stillerman ${ }^{17}$, Greg Wallace $^{17}$, Stephen Wukitch ${ }^{17}$, Lihua Zhou ${ }^{17}$, He Huang ${ }^{18}$, Kenneth Gentle ${ }^{18}$, Ken Liao ${ }^{18}$, Perry Philippe $^{18}$, William L. Rowan ${ }^{18}$, Linjin Zheng ${ }^{18}$, Patrick H. Diamond ${ }^{19}$, George R.Tynan ${ }^{19}$, Nicolas Fedorczak $^{19}$, Peter Manz ${ }^{19}$, Lei Zhao ${ }^{19}$, David Brower $^{20}$, Weixing Ding ${ }^{20}$, William W. Heidbrink ${ }^{21}$, Yubao Zhu ${ }^{21}$, Calvin W. Domier ${ }^{22}$, Neville C. Luhmann ${ }^{22}$, Xueqiao Xu ${ }^{23}$, Eric Wang ${ }^{23}$, Max E. Fenstermarcher ${ }^{23}$, Donald L. Hillis ${ }^{24}$, Rajesh Maingi ${ }^{24}$, Steve Meitner ${ }^{24}$, Igor V. Vinyar ${ }^{25}$, Vladimir Davydenko $^{26}$, Igor Shikhovtsev ${ }^{26}$, Naoko Ashikawa ${ }^{27}$, Kasahara Hiroshi ${ }^{27}$, Katsumi Ida ${ }^{27}$, Shinichiro Kado $^{27}$, Tomita Kawamura ${ }^{27}$, Saito Kenji ${ }^{27}$, Ryuhei Kumazawa ${ }^{27}$, Ogawa Kunihiro ${ }^{27}$, Isobe Mitsutaka $^{27}$, Shigeru Morita ${ }^{27}$, Haruhisa Nakano ${ }^{27}$, Satoshi Ohdachi ${ }^{27}$, Masaki Osakabe ${ }^{27}$, Mizúki Sakamoto ${ }^{27}$, Yasuhiko Takeiri ${ }^{27}$, Kazuo Toi ${ }^{27}$, Katsuyoshi Tsumori ${ }^{27}$, Nobuta Yuji ${ }^{27}$, Masaya Hanada $^{28}$, Mitsuru Kikuchi ${ }^{28}$, Atsushi Kojima ${ }^{28}$, Kazuhiro Watanabe ${ }^{28}$, Jean-Francois Artaud ${ }^{29}$, Vincent Basiuk ${ }^{29}$, F. Bouquey ${ }^{29}$, B. Bremond ${ }^{29}$, Gilles Coledani ${ }^{27}$, Laurent Colas ${ }^{29}$, Joan Decker ${ }^{29}$, D. Douai $^{29}$, Annika Ekedahl ${ }^{29}$, Christel Fenzi ${ }^{29}$, Eric Gauthier ${ }^{29}$, Gerardo Giruzzi ${ }^{29}$, Marc Goniche ${ }^{29}$, Dominique Guilhem ${ }^{29}$, Walid Helou ${ }^{29}$, Julien Hillairet ${ }^{29}$, Tuong Hoang ${ }^{29}$, Philippe Huynh ${ }^{29}$, Frederic Imbeaux $^{29}$, Xavier Litaudon ${ }^{29}$, Roland Magne ${ }^{29}$, Yves Peysson ${ }^{29}$, K. Vueillie $^{29}$, Xiaolan Zou ${ }^{29}$, Alberto Loarte $^{30}$, Manfred von Hellermann ${ }^{30}$, Franz Braun ${ }^{31}$, R. Bilato ${ }^{31}$, Volódymyr Bobkov ${ }^{31}$, J.M. Noterdaeme $^{31}$, Qingquan $\mathrm{Yu}^{31}$, Yunfeng Liang ${ }^{32}$, Jonny Pearson ${ }^{32}$, Michael Rack ${ }^{32}$, John Fessey ${ }^{33}$, Yueqiang Liu ${ }^{33}$, Charles Monroe ${ }^{33}$, Stefan Schmuck ${ }^{33}$, Paul Trimble ${ }^{33}$, Tom Todd ${ }^{33}$, Cheonho Bae ${ }^{34}$, Jun-Gyo Bak ${ }^{34}$, Suk-Ho Hong ${ }^{34}$, Sangong Lee ${ }^{34}$, Bae Young Soon ${ }^{34}$, Oh Byung Hoon ${ }^{35}$, Chang Doo $\mathrm{Hee}^{35}$, Lee Kwang $\mathrm{Won}^{35}$, Luca Amicucci ${ }^{36}$, Giuseppe Calabro ${ }^{36}$, Silvio Ceccuzzi ${ }^{36}$, Roberto Cesario $^{36}$, Flavio Crisanti ${ }^{36}$, Edmondo Giovannozzi ${ }^{36}$, Giuseppe Ramogida ${ }^{36}$, Gianmaria. De Tommasi $^{36}$, Angelo Antonio Tuccillo ${ }^{36}$, Bruno Viola ${ }^{36}$, Raffaele Albanese ${ }^{37}$, Roberto Ambrosino ${ }^{37}$, Lucio Barbato ${ }^{37}$, Stefano Mastrostefano ${ }^{37}$, Alfredo Pironti ${ }^{37}$,Vincenzo Pericoli Ridolfini ${ }^{37}$, Rory Scannell $^{37}$, Fabio Villone ${ }^{37}$, Volker Naulin ${ }^{38}$, Anders H. Nielsen ${ }^{38}$, Roman Zagorsky ${ }^{39}$, Sandor Zoletnik ${ }^{40}$, Chijin Xiao ${ }^{41}$, B.Madsen ${ }^{42}$, M. Salewski ${ }^{42}$

${ }^{1}$ Institute of Plasma Physics, Chinese Academy of Sciences, Hefei 230031, China

${ }^{2}$ University of Science and Technology of China, Hefei 230026, China

${ }^{3}$ Southwestern Institute of Physics, Chengdu 610041, China

${ }^{4}$ Tsinghua University, Beijing, China

${ }^{5}$ Peking University, Beijing, Chiń

${ }^{6}$ Zejiang University, Hangzhou, China

${ }^{7}$ Harbin Institute of Technology, Harbin 150001, China

${ }^{8}$ Huazhong University of Science and Technology, Wuhan, China

${ }^{9}$ Donghua University, Shanghai, China

${ }^{10}$ Dalian University of Technology, Dalian 116024, China

${ }^{11}$ Soochow University, Shuzhou, China

${ }^{12}$ China Academy of Engineering Physics, Mianyang 621900, China

${ }^{13}$ Hefei University of Technology, Hefei 230009, China

${ }^{14}$ Anhui University of Science and Technology, Huainan 232001, China

${ }^{15}$ General Atomic, San Diego, CA 92186-5608, USA

${ }^{16}$ Princeton Plasma Physics Laboratory, PO Box 451, Princeton, NJ 08543, USA

${ }^{17}$ Massachusetts Institute of Technology, Plasma Science and Fusion Center, Cambridge, MA 02139, USA

${ }^{18}$ Fusion Research Center, University of Texas at Austin, Austin, TX 78712, USA

${ }^{19}$ University of California, San Diego, CA 92093, USA

${ }^{20}$ University of California Los Angeles, Los Angeles, CA 90095, USA

${ }^{21}$ University of California at Irvine, Irvine, CA 92697, USA

${ }^{22}$ University of California at Davis, Davis, CA 95616, USA

${ }^{23}$ Lawrence Livermore National Laboratory (LLNL), Livermore, CA 94551, USA

${ }^{24}$ Oak Ridge National Laboratory, Oak Ridge, TN 37831-6169, USA

${ }^{25}$ PELIN, LLC, 27A, Gzhatskaya, Saint Petersburg 195220, Russia 
${ }^{26}$ Budker Institute of Nuclear Physics, Novosibirsk 630090, Russia

${ }^{27}$ National Institute for Fusion Sciences, Toki 509-5292, Japan

${ }^{28}$ Japan Atomic Energy Research Institute, Naka-machi, Naka-gun, Ibaraki-ken 311-0193, Japan

${ }^{29}$ CEA Cadarache, IRFM, F-13108 Saint Paul-lez-Durance, France

${ }^{30}$ ITER Organization, Route de Vinon sur Verdon, 13115 St Paul Lez Durance, France

${ }^{31}$ Max-Planck Institute for Plasma Physics, D-85748, Garching, Germany

${ }^{32}$ Association EURATOM-FZJ, D-52425 Jülich, Germany

${ }^{33}$ Euratom/CCFE Fusion Association, Culham Science Centre, Abingdon, Oxon. OX14 3DB, UK

${ }^{34}$ National Fusion Research Institute, Yuseong-Gu, Daejeon, 305-806, Korea

${ }^{35}$ Korea Atomic Energy Research Institute,Yuseong-Gu, Daejeon, 305-353,Korea

${ }^{36}$ ENEA Unità Tecnica Fusione, C.R. Frascati, Via E. Fermi 45, 00044 Frascati, Roma, Italy

${ }^{37}$ CREATE, Università di Napoli Federico II, Università di Cassino and Università di Napoli

Parthenope, Via Claudio 19, 80125 Napoli, Italy

${ }^{38}$ Association Euratom-Risø DTU, Roskilde, Denmark

${ }^{39}$ Institute of Plasma Physics and Laser Microfusion, Warsaw, Poland

${ }^{40}$ Association EURATOM - KFKI RMKI, P.O. Box 49, H-1525 Budapest, Hungary

${ }^{41}$ University of Saskatchewan, Saskatchewan, S7N 5E5, Canada

${ }^{42}$ Department of physics, Technical University of Denmark, Kgs. Lyngby, Denmark 
[16] Porkolab M, Bernabei S, Hooke W M, Motley R W and Nagashima T 1977 Observation of parametric instabilities in lower-hybrid radio-frequency heating of tokamaks Phys. Rev. Lett. 38 230-3

[17] Baek S G, Părker R R, Shiraiwa S, Wallace G M, Bonoli P T, Brunner D, Faust I C, Hubbard A E, Labombard B and Porkolab M 2013 Measurements of ion cyclotron parametric decay of lower hybrid waves at the high-field side of Alcator C-Mod Plasma Phys. Control. Fusion 55

[18] Goniche M, Basiuk V, Decker J, Sharma P K, Antar G, Berger-By G, Clairet F, Delpech L, Ekedahl A, Gunn J, Hillairet J, Litaudon X, Mazon D, Nilsson E, Oosako T, Peysson Y, Preynas M, Prou M and Ségui J L 2013 Lower hybrid current drive at high density on Tore Supra Nucl. Fusion 53

[19] Cesariô R, Amicucci L, Cardinali A, Castaldo C and Tuccillo A A 2011 Lower hybrid current drive at plasma densities required for thermonuclear reactors AIP Conf. Proc. 1406 423-6

[20] Ding B J, Li Y C, Zhang L, Li M H, Wei W, Kong E H, Wang M, Xu H D, Wang S L, Xu G S, Zhao L M, Hu H C, Jia H, Cheng M, Yang Y, Liu L, Zhao H L, Peysson Y, Decker J, Goniche M, Amicucci L, Cesario R, Tuccillo 
[33] Zhang L, Morita S, Xu Z, Zhang P F, Zang Q, Duan Y M, Liu H Q, Zhao H L, Ding F, Ohishi T, Gao W, Huang J, Yang X D, Chen Y J, Wu Z W, Xu P, Ding B J, Hu C D, Gong X Z, Chen J L and Hu L Q 2017 Suppression of tungsten accumulation during ELMy H-mode by lower hybrid wave heating in the EAST tokamak Nucl. Mater. Energy 12 774-8

[34] Wu K, Yuan Q P, Xiao B J, Wang L, Duan Y M, Chen J B, Zheng X W, Liu X J, Zhang B, Xu J C, Luo Z P, Zang Q, Li Y Y, Feng W, Wu J H, Yang Z S, Zhang L, Luo G N, Gong X Z, Hu L Q, Hu J S and Li J 2018 Achievement of radiative feedback control for long-pulse operation on EAST Nucl. Fusion 58

[35] Yang Z, Chen J, Coster D P, Duan Y, Wang L, Ding F, Xu J, Zang Q, Wang T, Yan N, Zhang T, Zhang L, Wu J, Peng Y K M and Luo G N 2017 Modeling of radiative divertor experiments with argon seeding for H-mode plasma in EAST Phys. Plasmas 24

[36] Xu G S 2018 A PROMISING GRASSY ELM REGIME FOR HIGH-PERFORMANCE STEADY- STATE OPERATIONS WITH METAL WALL IN EAST AND CFETR 27th IAEA FEC EX/P2-4

[37] Chen L, Xü G S, Yan N, Nielsen A H, Liu J B, Gao W, Chen R, Qu H, Ding F, Mao H M, Sun Z, Zhang L, Li M H, Zhang W, Hu G H, Shao L M, Wu X Q, Du H F, Xu J C, Li Y L, Zhao N, Lan H, Ye Y, Zhang H, Wang H Q, Ding S Y, Wang L and Wan B N 2018 Effect of $\nabla$ B drift on the H-mode power threshold in upper single null plasmàs with ITER-like tungsten divertor on EAST Phys. Plasmas 25

[38] Yu Y, Wang L, Cao B, Wang H and Hu J 2017 Fuel retention and recycling studies by using particle balance in EAST tokamak 


\section{List of figure captions}

Fig. 1 Time histories of plasma current, loop voltage, electron density, RF heating power of LHW, ECH and ICRF, divertor temperature by IR camera, radiation power, confinement factor $\mathrm{H}_{98 \mathrm{y} 2}$, and $\mathrm{D} \alpha$ (from top to bottom).

Fig. 2 Electron temperature, density profiles by TS, transport coefficient and electron heating power profiles for discharge 73999

Fig. 3 Normalized poloidal beta versus line-averaged density of low loop voltage plasma

Fig. 4 Time history of several parameters for high $\beta \mathrm{P}$ discharges. Left from top to bottom, normalized poloidal beta \& normalized beta, loop voltage \& line averaged density over Greenwald density limit, LHW\&ECH power; Right from top to bottom, normalized poloidal beta \& normalized beta \& loop voltage, LHW\&ECH power, NB power

Fig. 5 High $\beta_{N}$ scenario development for EAST\#78723 with $\beta_{N}>1.9$ sustained for 2 s. Signals from top to bottom are plasma current (Ip) and loop voltage, LHW power (PLHW) and NBI power (PNBI), the core line averaged density, plasma normalized beta and inductance.

Fig.6 Operational regime of the high $\beta_{\mathrm{N}}$ scenario, where the $\beta_{\mathrm{N}}$ value has reached 3 times of $l_{i}$.

Fig.7 An example of $T_{i}$ profiles before and after ITB formation

Fig.8 Current profile measured by POINT and frequency spectra measured by RF probe with different LH frequencies (left) and densities (right).

Fig.9 Normalized experimental current drive efficiency versus pump spectral width. Here, the pump width $\Delta \mathrm{f}_{\mathrm{P}}$ is defined as the full width $20 \mathrm{db}$ below the maximum.

Fig. 10. Time evolution of ECRH heating power, energy confinement $\mathrm{H}_{98 \mathrm{y} 2}$ and internal inductance $l_{i}$ of EAST shot \#66743. ECRH is turned off at 3.91s.

Fig.11 LHW driven current profiles before and after ECRH termination calculated by GENRAY and CQL3D codes.

Fig. 12 Statistics of ELM frequency as a function of $q_{95}, \beta_{\mathrm{p}}, \mathrm{n}_{\mathrm{e}} / \mathrm{n}_{\mathrm{GW}}$, upper triangularity $\delta_{\mathrm{u}}$ and LHCD power $\mathrm{P}_{\mathrm{LHCD}}$ for EAST $\mathrm{H}$-mode discharges with the plasma stored energy $\mathrm{W}_{\mathrm{p}}$ $>120 \mathrm{~kJ}$, indicating the access parameter space of the high-frequency small-ELM regime ( $f_{\mathrm{ELM}}>0.5 \mathrm{kHz}$ ) is $q_{95} \geq 5.3, \beta_{\mathrm{p}} \geq 1.1$ and $\mathrm{n}_{\mathrm{e}} / \mathrm{n}_{\mathrm{GW}} \geq 0.46$. High upper triangularity $\delta_{\mathrm{u}}$ appears to be beneficial for access to this regime. In addition, access to this regime appears to be independent of the LHCD power. The magenta curves indicate the lower boundaries of the regime access for these parameters.

Fig. 13 ELM suppression achieved in a large q95 window ranging from 3.2 to 4.2 in EAST. Here the $n=2$ RMP with a coil current $2.9 \mathrm{kA}$ has been applied from $3.5 \mathrm{~s}$ to $6.5 \mathrm{~s}$.

Fig. 14 Time histories of various plasma quantities for a H-mode plasma discharge \#55251 on EAST during the application of periodically alternating neutral beam injection. (a) LHW power $P_{L H W}(2.45 \mathrm{GHz}$ and $4.6 \mathrm{GHz})$ and ICRF power $P_{I C R F}$, (b) line averaged density $n_{e}$ and stored energy $W_{E}$, (d) co- and countercurrent NBI power $P_{N B I}$, (e) (f) the density of particle flux $\Gamma_{\text {ion }}$ at the divertor target (black solid line) and the velocity of toroidal rotation of the central plasma $v_{t}$ (red dash line).

Fig. 15 Radial profiles of Doppler shift $f_{\text {Doppler }}$ (here, $E_{r} \propto f_{\text {Doppler }}$ ) with co- and counter-current NBL in H-mode discharge, respectively. The blue line is profiles in Lmode discharge.

Fig.16 Time evolution of (a) injected power of 4.6GHz LHW, ICRH and ECRH (b) divertor $\mathrm{D}_{\alpha}$, (c) ne normalized intensity of emission line of Mo XXXII at $127.87 \AA$ and W-UTA in the range of 45-70 $\AA$ (composed of $\mathrm{W}^{27+}-\mathrm{W}^{45+}$ ), (d) Impurity concentration of Mo and W Right: Density profile of $\mathrm{W}^{45+}$ ion with (red square) and without (blue circle) on-axis ECRH 
Fig. 17 The time traces of three sequential H-mode discharges for radiative feedback control with different target radiated power ( $\mathrm{P}_{\text {rad,target }}$ ): (a) 0.6 MW without a feedforward Ne injection, (b) $0.8 \mathrm{MW}$, (c) $1.0 \mathrm{MW}$ with the IR-camera measured temperature for the upper outer divertor plate, (d) the contour of the temperature measured by the IR camera for the upper outer divertor target plate in the same shot with figure (c), with the vertical axis being the distance along the target plate poloidally.

Fig.18 (a) HWP plasmas under 1T, (b) DC-GDC plasmas under $2 \mathrm{~T}$ with 4 anodes working and $5 \mathrm{~A}$ /anode.

Fig.19 The path to the goal of $\mathrm{f}_{\mathrm{bs}} \sim 50 \%$, based on the $0 \mathrm{D}$ simulation for Ip=400 kA plasma operation. Color bar shows the line-averaged density in each case. The stars are two typical discharges in EAST campaign 2018. The red ellipse shows the collection of the long-pulse regime in 2017, which is also the start point of this extrapolation.

Fig.20 EAST new W lower divertor. Installation scheduled in 2019. 
Fig.1

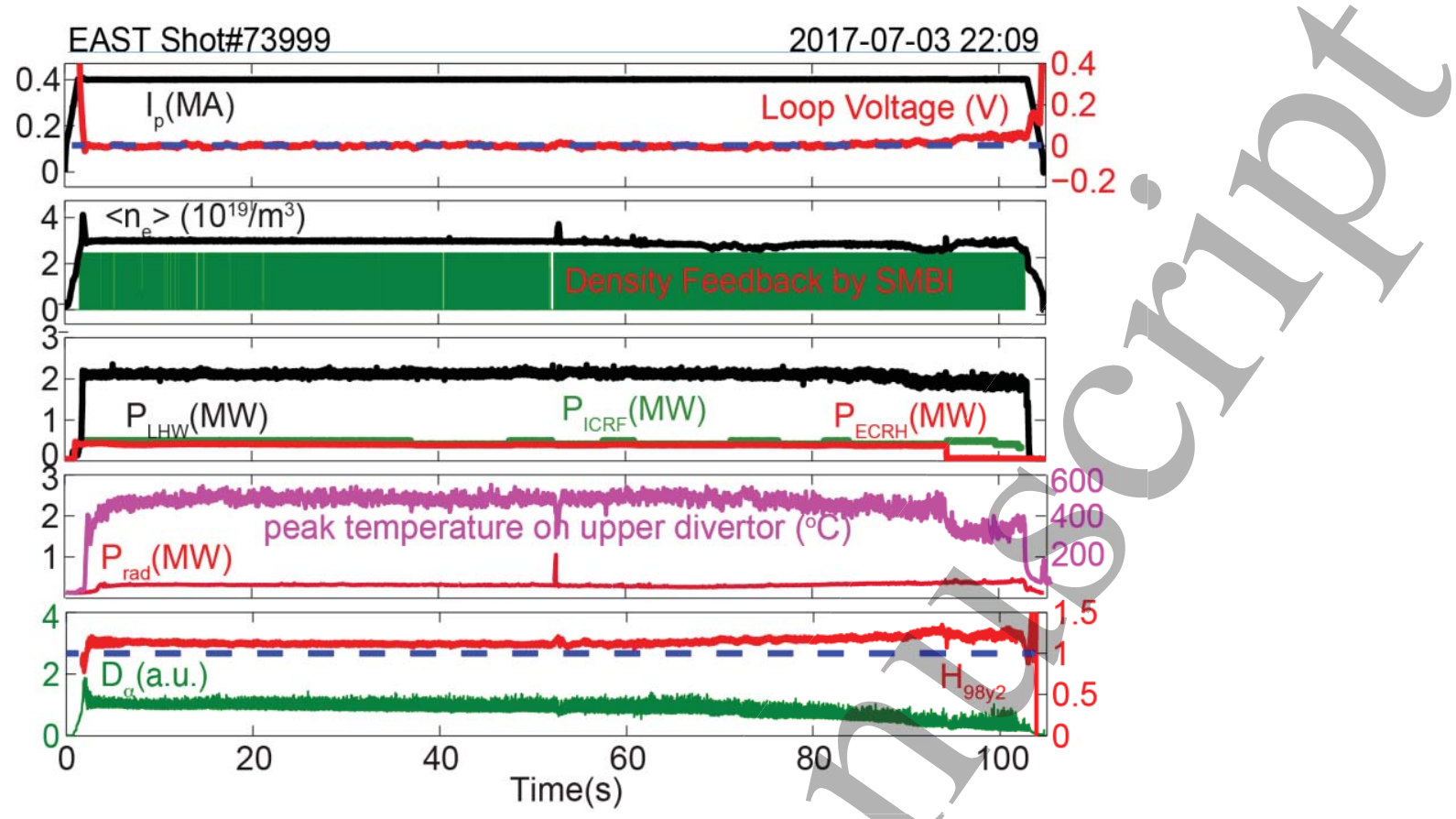


Fig.2
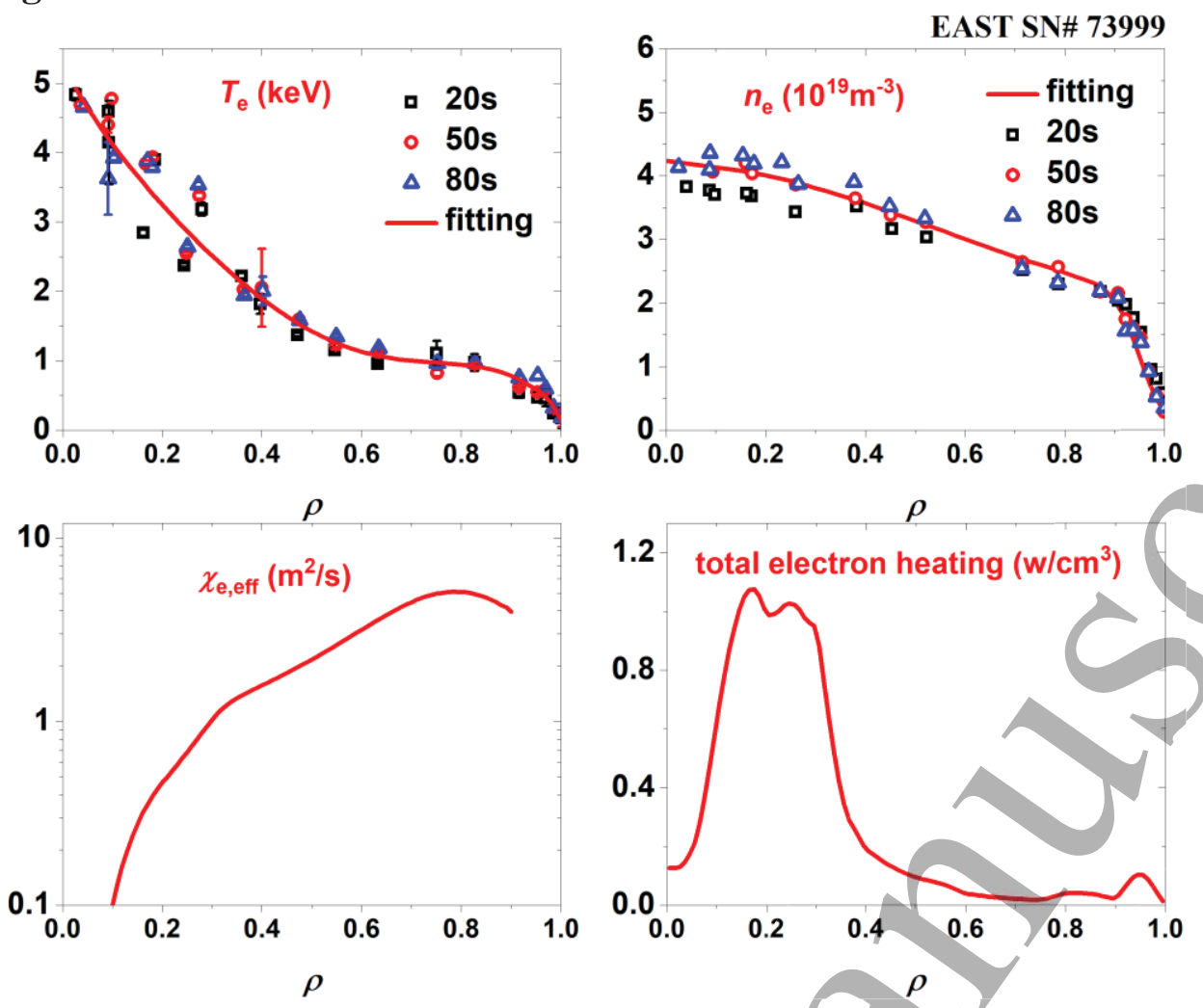


\section{Fig.3}

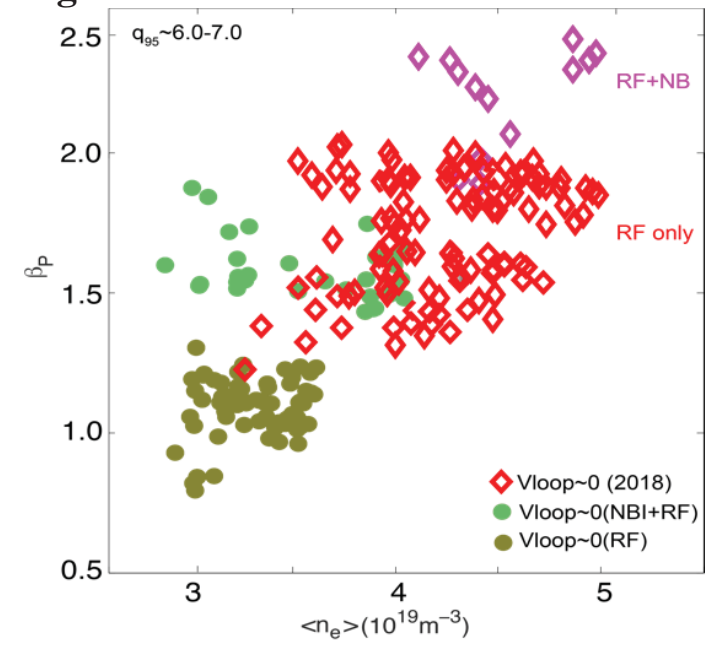


Fig. 4
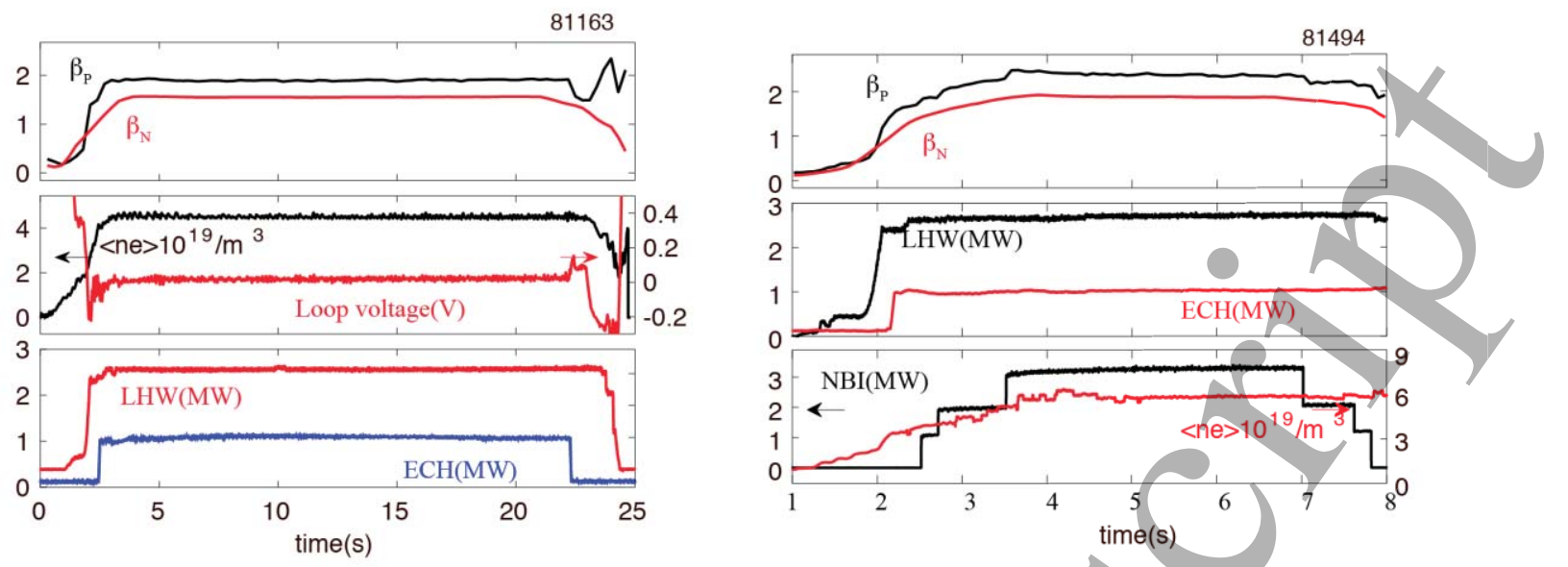


\section{Fig.5}

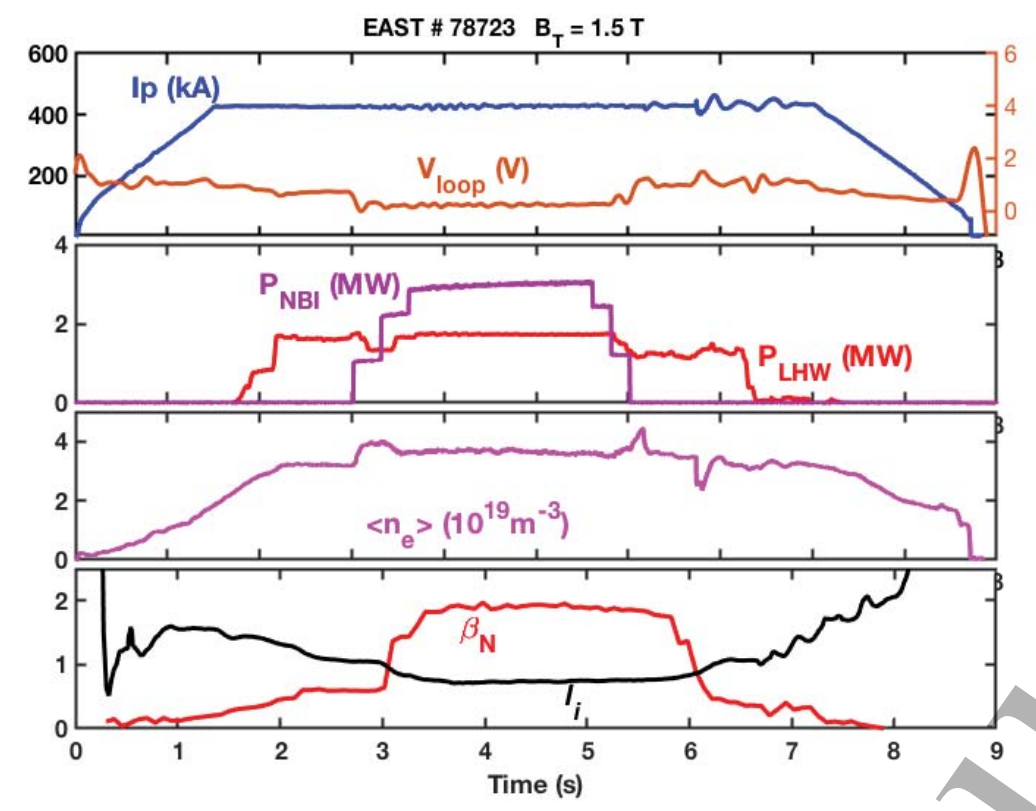




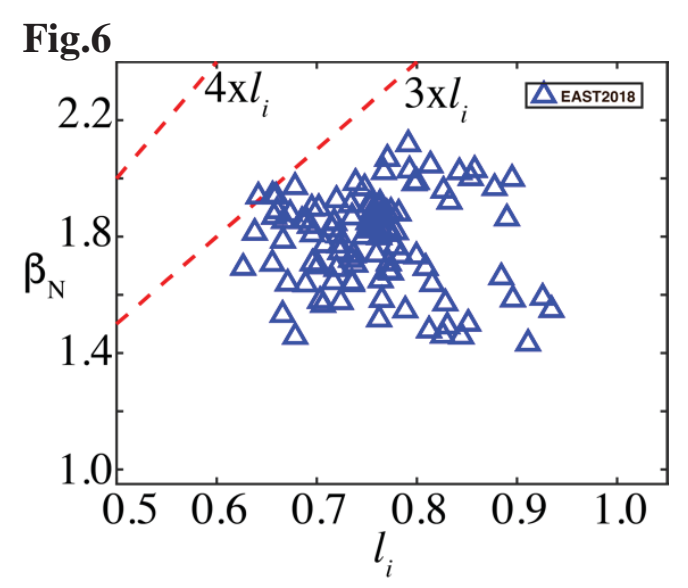

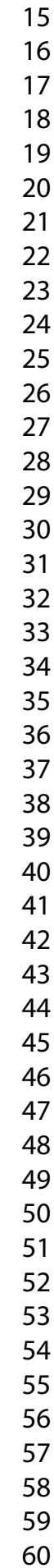


Fig.7

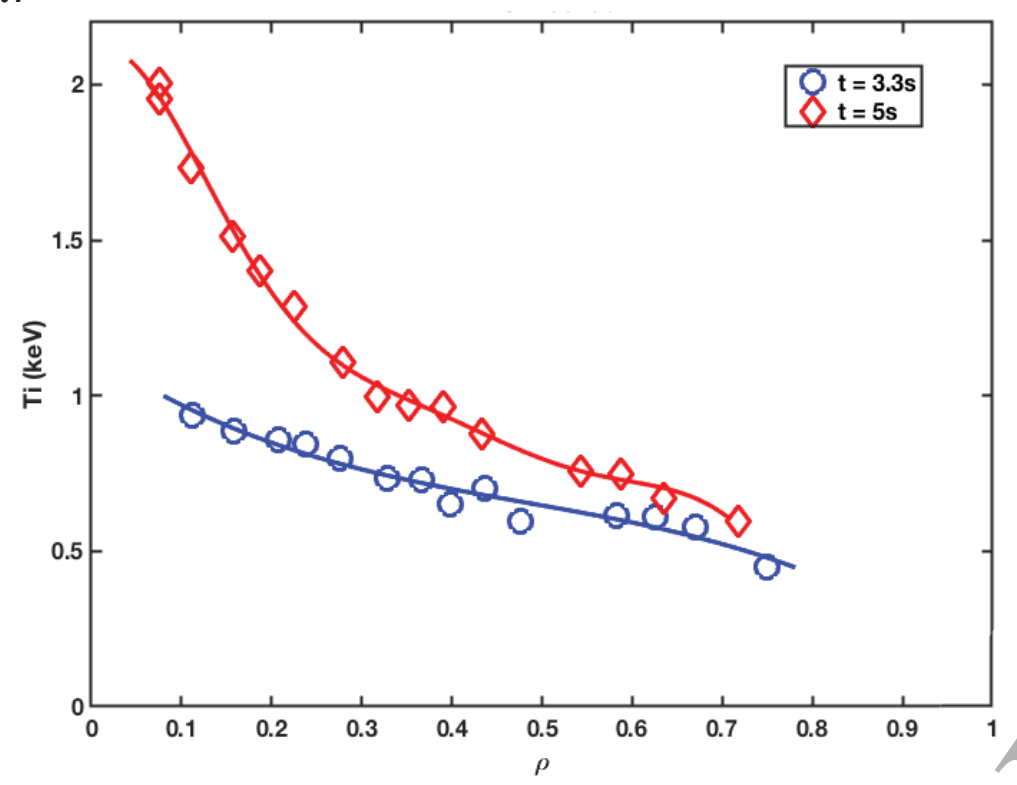




\section{Fig.8}

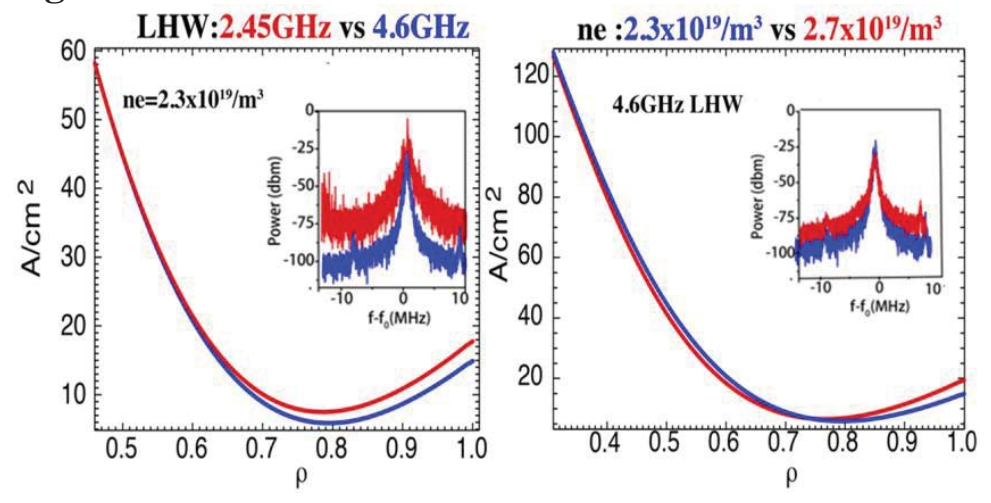


Fig.9

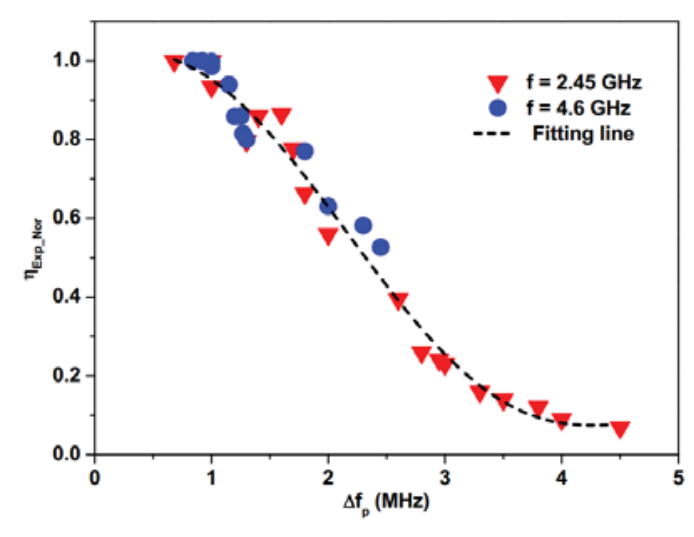


Fig.10

\section{EAST SN\#66743}

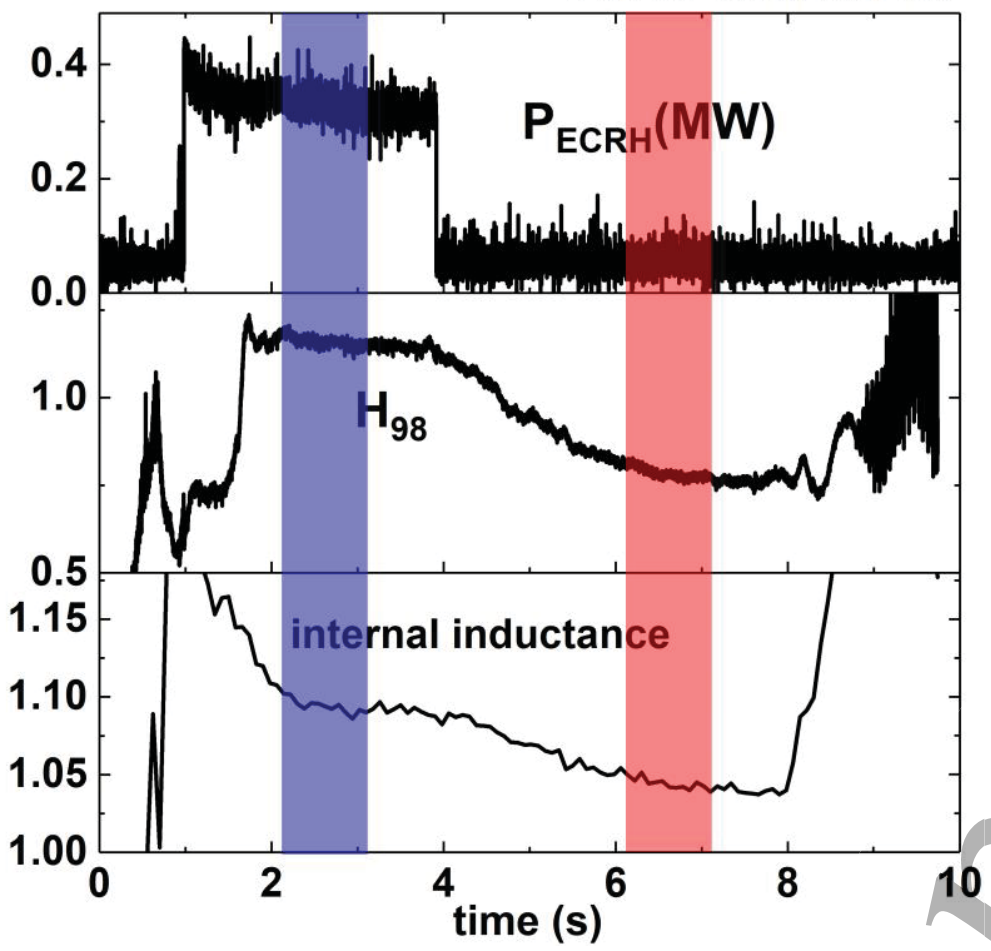




\section{Fig.11}

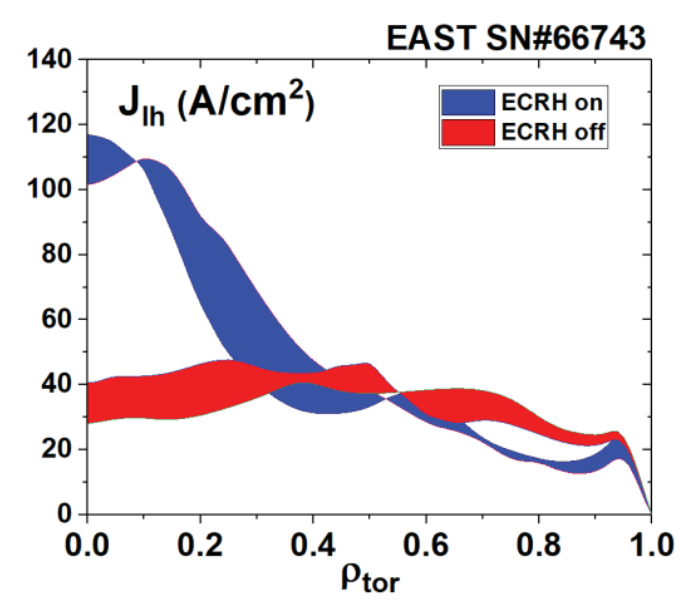


Fig.12
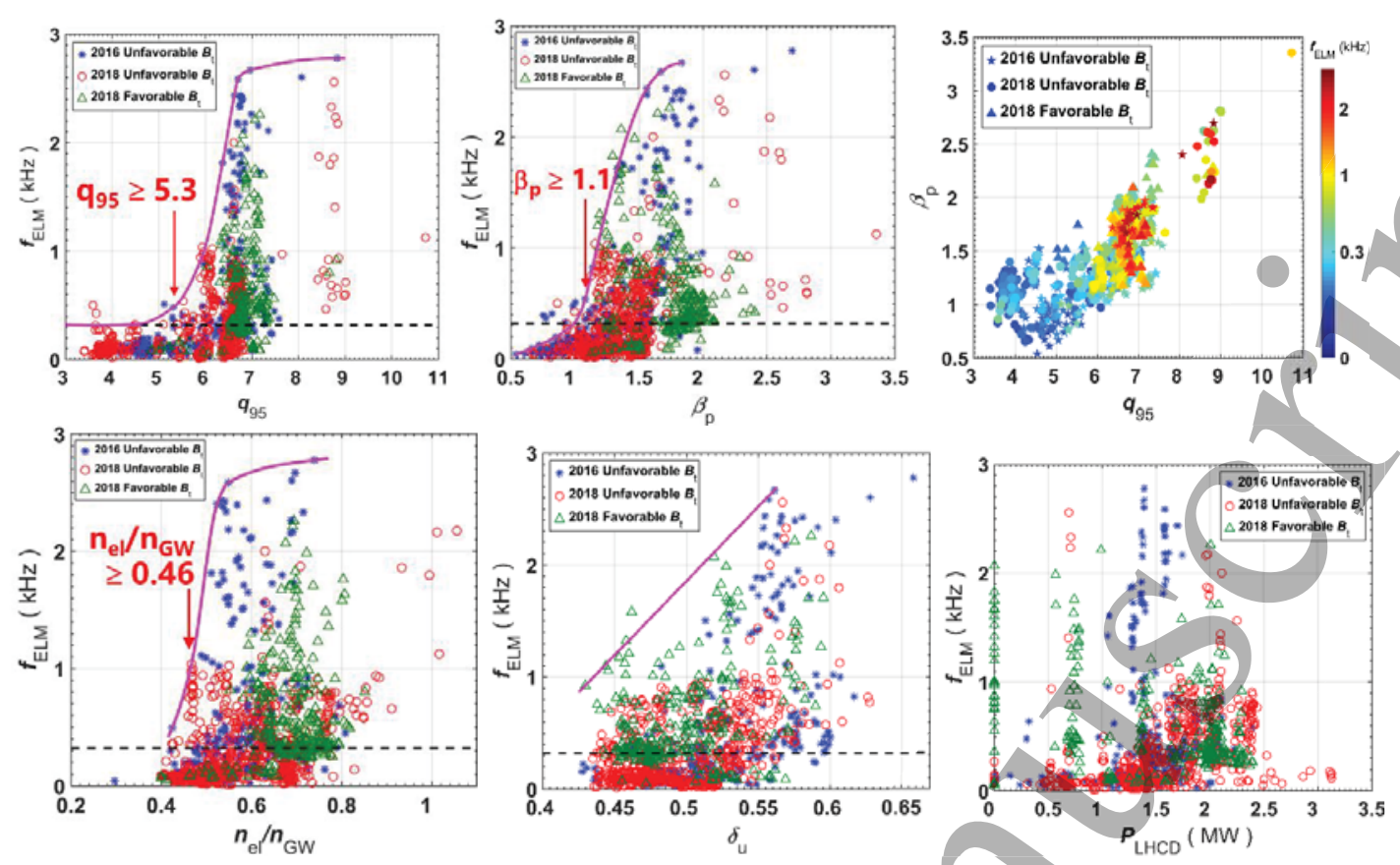
Fig.13

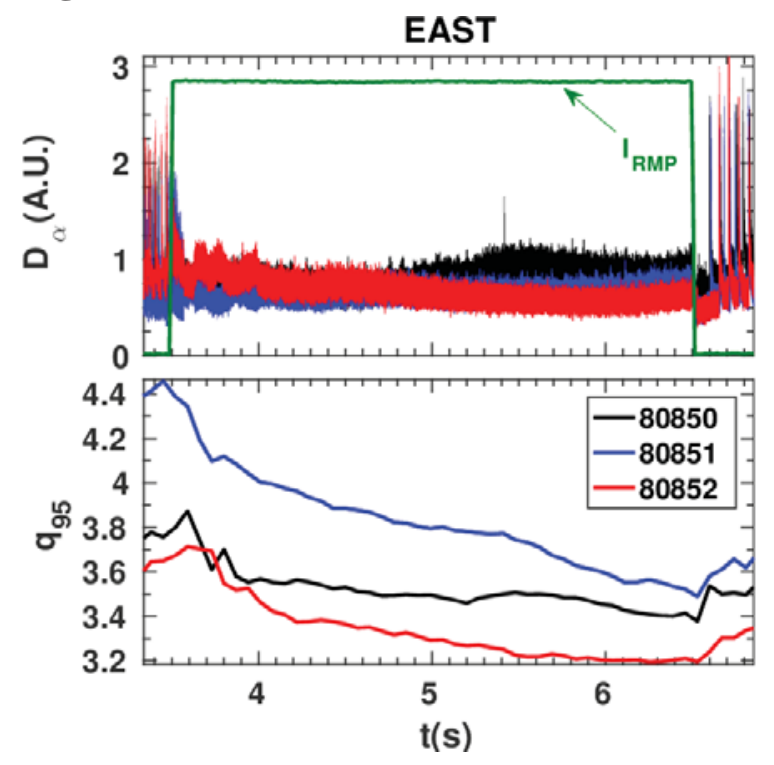


Fig.14
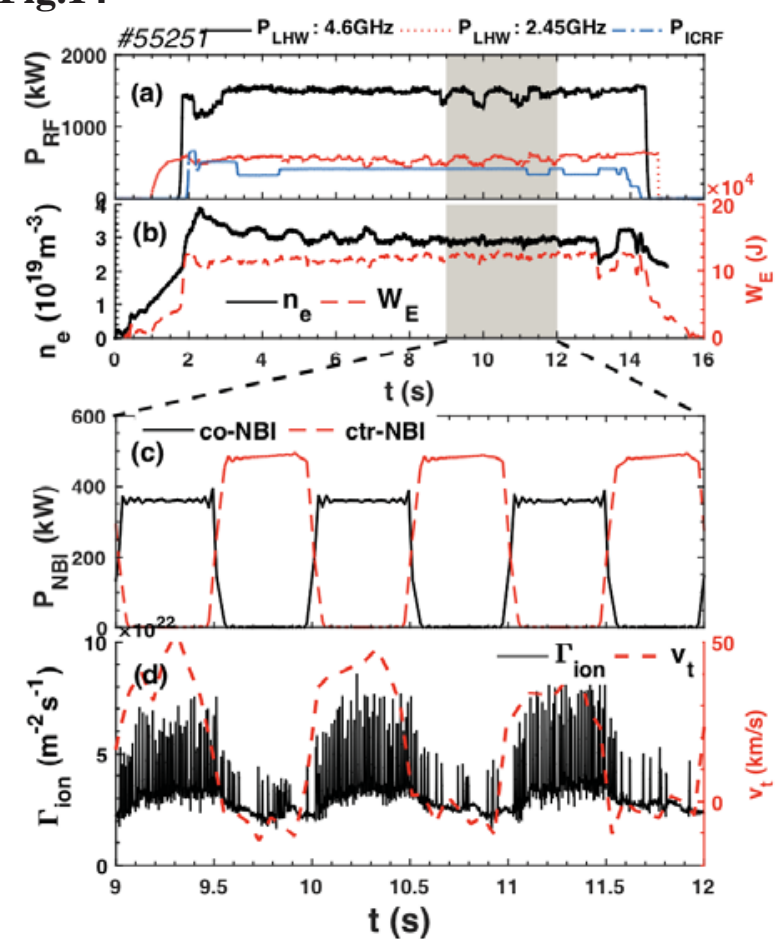


\section{Fig.15}

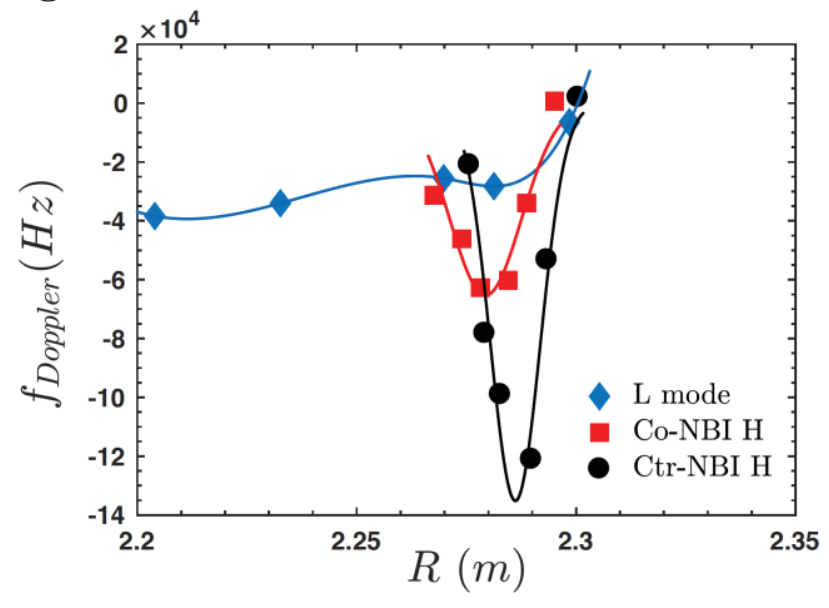




\section{Fig.16}
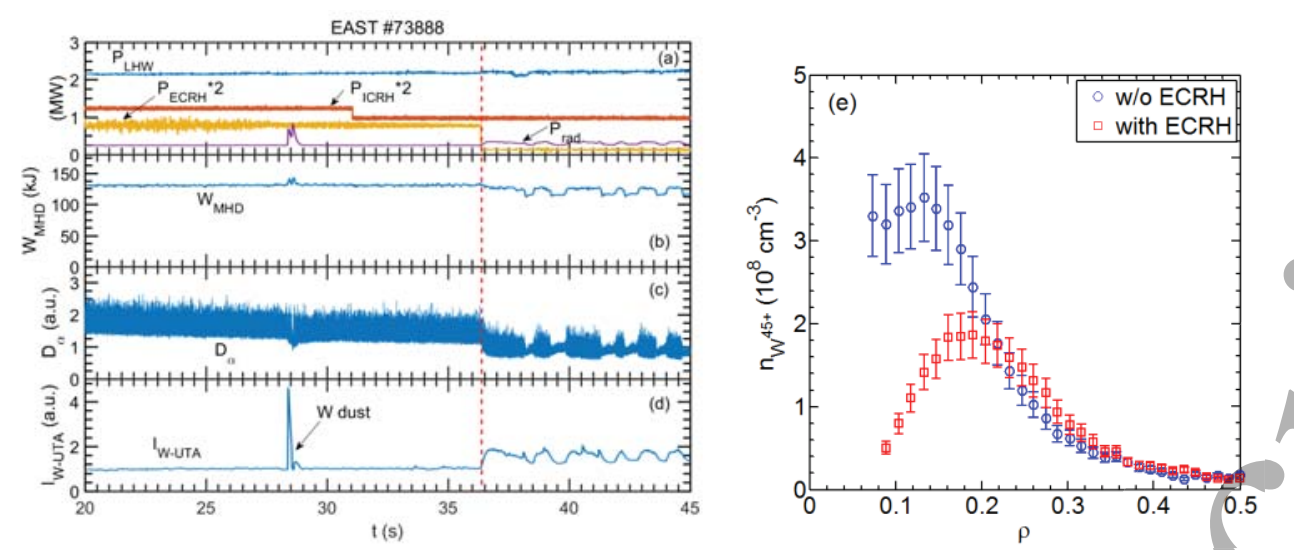
Fig.17
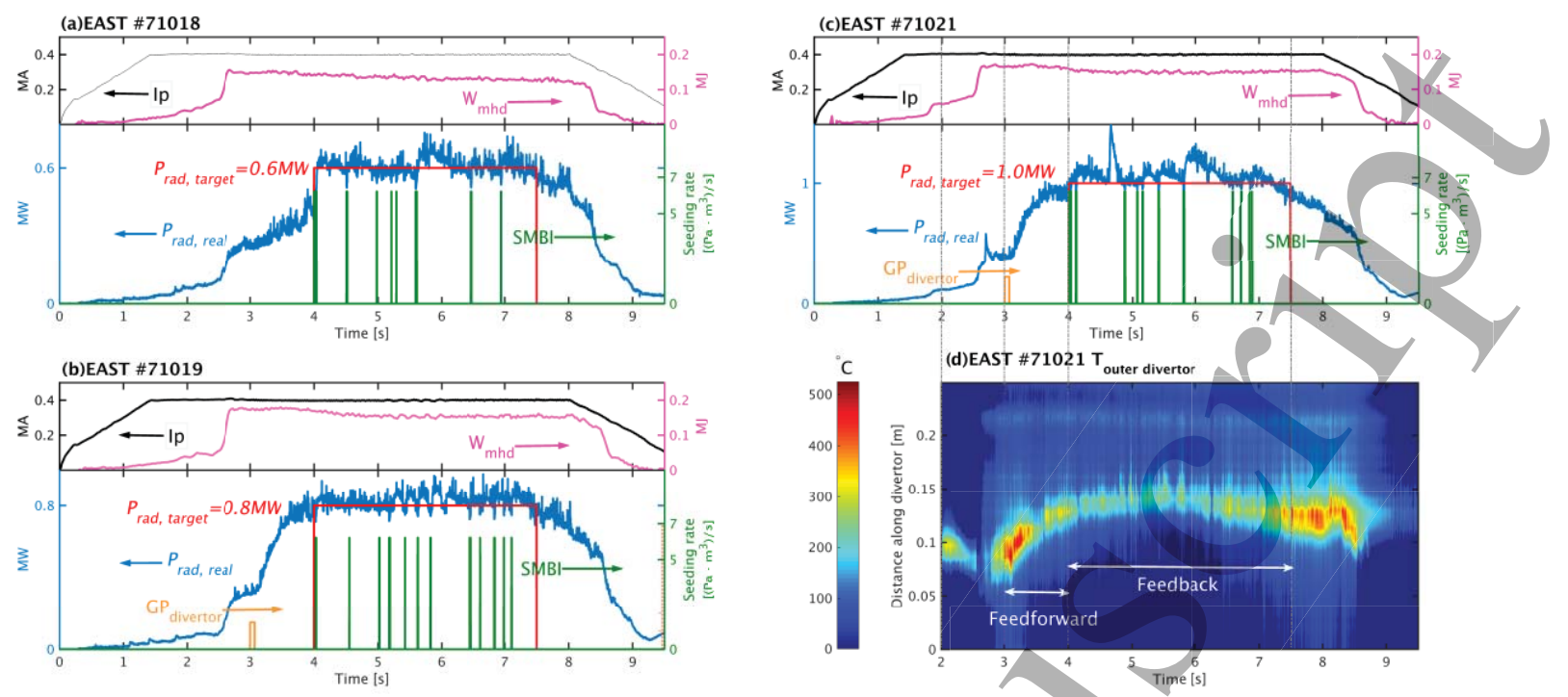
Fig. 18

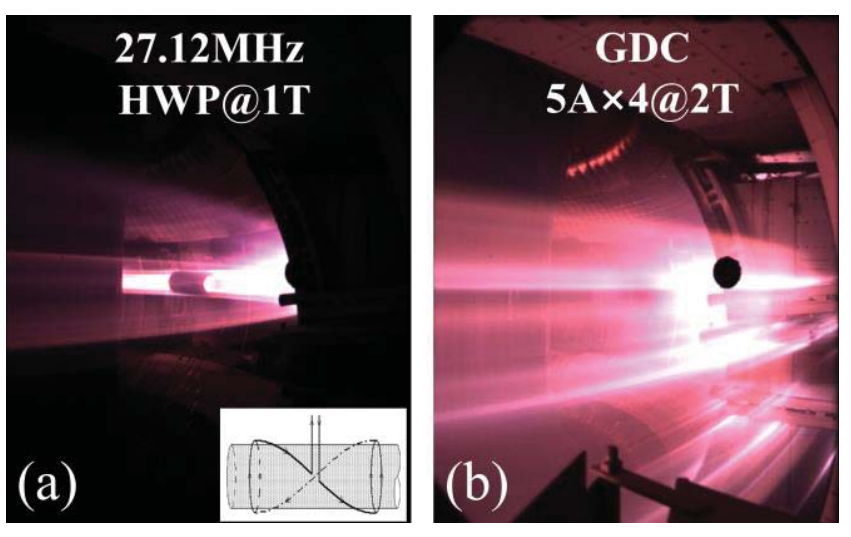


Fig.19

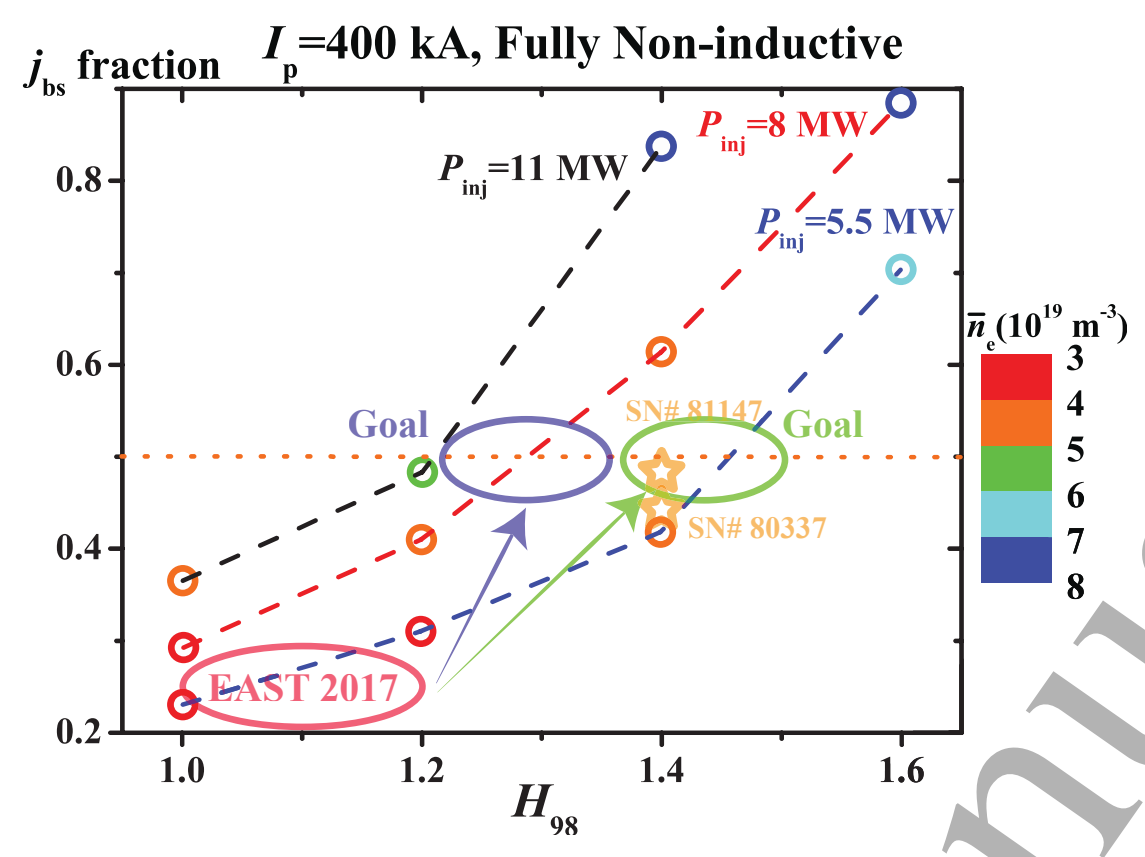


Fig. 20

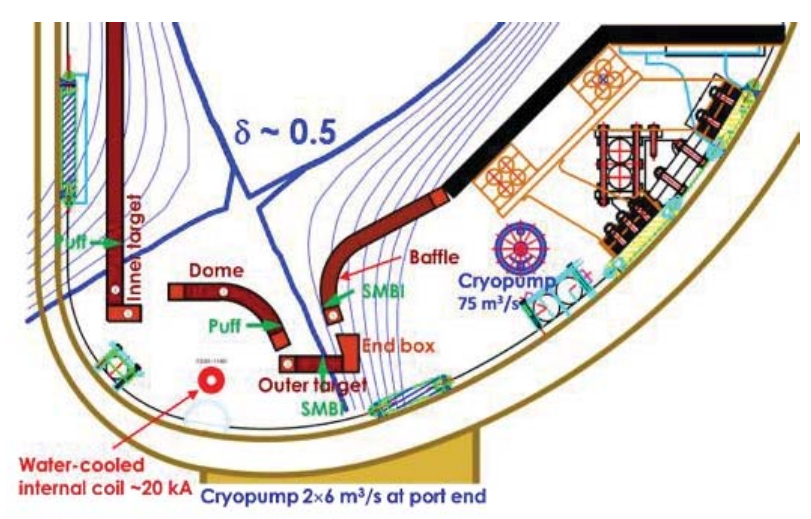

